\title{
The Thermodynamics of Black Holes
}

\author{
Robert M. Wald \\ Enrico Fermi Institute and Department of Physics \\ University of Chicago \\ 5640 S. Ellis Avenue \\ Chicago, Illinois 60637-1433 \\ email:rmwa@midway.uchicago.edu \\ http://physics.uchicago.edu/t_rel.html\#Wald \\ Published on 9 July 2001 \\ www.livingreviews.org/Articles/Volume4/2001-6wald \\ Living Reviews in Relativity \\ Published by the Max Planck Institute for Gravitational Physics \\ Albert Einstein Institute, Germany
}

\begin{abstract}
We review the present status of black hole thermodynamics. Our review includes discussion of classical black hole thermodynamics, Hawking radiation from black holes, the generalized second law, and the issue of entropy bounds. A brief survey also is given of approaches to the calculation of black hole entropy. We conclude with a discussion of some unresolved open issues.
\end{abstract}

(C)2001 Max-Planck-Gesellschaft and the authors. Further information on copyright is given at http://www.livingreviews.org/Info/Copyright/. For permission to reproduce the article please contact livrev@aei-potsdam.mpg.de. 


\section{Article Amendments}

On author request a Living Reviews article can be amended to include errata and small additions to ensure that the most accurate and up-to-date information possible is provided. For detailed documentation of amendments, please go to the article's online version at

http://www.livingreviews.org/Articles/Volume4/2001-6wald/.

Owing to the fact that a Living Reviews article can evolve over time, we recommend to cite the article as follows:

Wald, R.M.,

"The Thermodynamics of Black Holes",

Living Rev. Relativity, 4, (2001), 6. [Online Article]: cited on <date>, http://www.livingreviews.org/Articles/Volume4/2001-6wald/.

The date in 'cited on <date>' then uniquely identifies the version of the article you are referring to. 


\section{Contents}

11 Introduction 4

2 Classical Black Hole Thermodynamics 6

3 Hawking Radiation 12

4 The Generalized Second Law (GSL) 16

4.1 Arguments for the validity of the GSD $\ldots \ldots \ldots \ldots$

1.2 Entropy bounds . . . . . . . . . . . . . . . . . . . . . . . . . . . 19

5 Calculations of Black Hole Entropy 24

6 Open Issues 29

6.1 Does a pure quantum state evolve to a mixed state in the process of black hole formation and evaporation? . . . . . . . . . . . . . 29

6.2 What (and where) are the degrees of freedom responsible for black hole entropy? . . . . . . . . . . . . . . . . . 31

7 Acknowledgements 33

$\begin{array}{ll}\text { References } & 34\end{array}$ 


\section{Introduction}

During the past 30 years, research in the theory of black holes in general relativity has brought to light strong hints of a very deep and fundamental relationship between gravitation, thermodynamics, and quantum theory. The cornerstone of this relationship is black hole thermodynamics, where it appears that certain laws of black hole mechanics are, in fact, simply the ordinary laws of thermodynamics applied to a system containing a black hole. Indeed, the discovery of the thermodynamic behavior of black holes - achieved primarily by classical and semiclassical analyses - has given rise to most of our present physical insights into the nature of quantum phenomena occurring in strong gravitational fields.

The purpose of this article is to provide a review of the following aspects of black hole thermodynamics:

- At the purely classical level, black holes in general relativity (as well as in other diffeomorphism covariant theories of gravity) obey certain laws which bear a remarkable mathematical resemblance to the ordinary laws of thermodynamics. The derivation of these laws of classical black hole mechanics is reviewed in section $\square$.

- Classically, black holes are perfect absorbers but do not emit anything; their physical temperature is absolute zero. However, in quantum theory black holes emit Hawking radiation with a perfect thermal spectrum. This allows a consistent interpretation of the laws of black hole mechanics as physically corresponding to the ordinary laws of thermodynamics. The status of the derivation of Hawking radiation is reviewed in section 3 .

- The generalized second law (GSL) directly links the laws of black hole mechanics to the ordinary laws of thermodynamics. The arguments in favor of the GSL are reviewed in section 6 . A discussion of entropy bounds is also included in this section.

- The classical laws of black hole mechanics together with the formula for the temperature of Hawking radiation allow one to identify a quantity associated with black holes - namely $A / 4$ in general relativity - as playing the mathematical role of entropy. The apparent validity of the GSL provides strong evidence that this quantity truly is the physical entropy of a black hole. A major goal of research in quantum gravity is to provide an explanation for - and direct derivation of - the formula for the entropy of a black hole. A brief survey of work along these lines is provided in section 15 .

- Although much progress has been made in our understanding of black hole thermodynamics, many important issues remain unresolved. Primary among these are the "black hole information paradox" and issues related to the degrees of freedom responsible for the entropy of a black hole. These unresolved issues are briefly discussed in section [6.

Living Reviews in Relativity (2001-6)

http://www.livingreviews.org 
Throughout this article, we shall set $G=\hbar=c=k=1$, and we shall follow the sign and notational conventions of [99]. Although I have attempted to make this review be reasonably comprehensive and balanced, it should be understood that my choices of topics and emphasis naturally reflect my own personal viewpoints, expertise, and biases.

Living Reviews in Relativity (2001-6)

http://www.livingreviews.org 


\section{Classical Black Hole Thermodynamics}

In this section, I will give a brief review of the laws of classical black hole mechanics.

In physical terms, a black hole is a region where gravity is so strong that nothing can escape. In order to make this notion precise, one must have in mind a region of spacetime to which one can contemplate escaping. For an asymptotically flat spacetime $\left(M, g_{a b}\right)$ (representing an isolated system), the asymptotic portion of the spacetime "near infinity" is such a region. The black hole region, $\mathcal{B}$, of an asymptotically flat spacetime, $\left(M, g_{a b}\right)$, is defined as

$$
\mathcal{B} \equiv M-I^{-}\left(\mathcal{I}^{+}\right),
$$

where $\mathcal{I}^{+}$denotes future null infinity and $I^{-}$denotes the chronological past. Similar definitions of a black hole can be given in other contexts (such as asymptotically anti-deSitter spacetimes) where there is a well defined asymptotic region.

The event horizon, $\mathcal{H}$, of a black hole is defined to be the boundary of $\mathcal{B}$. Thus, $\mathcal{H}$ is the boundary of the past of $\mathcal{I}^{+}$. Consequently, $\mathcal{H}$ automatically satisfies all of the properties possessed by past boundaries (see, e.g., [5.5] or [9.9] for further discussion). In particular, $\mathcal{H}$ is a null hypersurface which is composed of future inextendible null geodesics without caustics, i.e., the expansion, $\theta$, of the null geodesics comprising the horizon cannot become negatively infinite. Note that the entire future history of the spacetime must be known before the location of $\mathcal{H}$ can be determined, i.e., $\mathcal{H}$ possesses no distinguished local significance.

If Einstein's equation holds with matter satisfying the null energy condition (i.e., if $T_{a b} k^{a} k^{b} \geq 0$ for all null $k^{a}$ ), then it follows immediately from the Raychauduri equation (see, e.g., [9.9]) that if the expansion, $\theta$, of any null geodesic congruence ever became negative, then $\theta$ would become infinite within a finite affine parameter, provided, of course, that the geodesic can be extended that far. If the black hole is strongly asymptotically predictable - i.e., if there is a globally hyperbolic region containing $I^{-}\left(\mathcal{I}^{+}\right) \cup \mathcal{H}$ - it can be shown that this implies that $\theta \geq 0$ everywhere on $\mathcal{H}$ (see, e.g., [5.5, 9.9]). It then follows that the surface area, $A$, of the event horizon of a black hole can never decrease with time, as discovered by Hawking [5.3].

It is worth remarking that since $\mathcal{H}$ is a past boundary, it automatically must be a $C^{0}$ embedded submanifold (see, e.g., [9.9]), but it need not be $C^{1}$. However, essentially all discussions and analyses of black hole event horizons implicitly assume $C^{1}$ or higher order differentiability of $\mathcal{H}$. Recently, this higher order differentiability assumption has been eliminated for the proof of the area theorem [36].

The area increase law bears a resemblance to the second law of thermodynamics in that both laws assert that a certain quantity has the property of never decreasing with time. It might seem that this resemblance is a very superficial one, since the area law is a theorem in differential geometry whereas the second

Living Reviews in Relativity (2001-6)

http://www.livingreviews.org 
law of thermodynamics is understood to have a statistical origin. Nevertheless, this resemblance together with the idea that information is irretrievably lost when a body falls into a black hole led Bekenstein to propose [114, 1.5] that a suitable multiple of the area of the event horizon of a black hole should be interpreted as its entropy, and that a generalized second law (GSL) should hold: The sum of the ordinary entropy of matter outside of a black hole plus a suitable multiple of the area of a black hole never decreases. We will discuss this law in detail in section $\mathbb{\text { ⿴囗十 }}$.

The remaining laws of thermodynamics deal with equilibrium and quasiequilibrium processes. At nearly the same time as Bekenstein proposed a relationship between the area theorem and the second law of thermodynamics, Bardeen, Carter, and Hawking [ [I2] provided a general proof of certain laws of "black hole mechanics" which are direct mathematical analogs of the zeroth and first laws of thermodynamics. These laws of black hole mechanics apply to stationary black holes (although a formulation of these laws in terms of isolated horizons will be briefly described at the end of this section).

In order to discuss the zeroth and first laws of black hole mechanics, we must introduce the notions of stationary, static, and axisymmetric black holes as well as the notion of a Killing horizon. If an asymptotically flat spacetime $\left(M, g_{a b}\right)$ contains a black hole, $\mathcal{B}$, then $\mathcal{B}$ is said to be stationary if there exists a one-parameter group of isometries on $\left(M, g_{a b}\right)$ generated by a Killing field $t^{a}$ which is unit timelike at infinity. The black hole is said to be static if it is stationary and if, in addition, $t^{a}$ is hypersurface orthogonal. The black hole is said to be axisymmetric if there exists a one parameter group of isometries which correspond to rotations at infinity. A stationary, axisymmetric black hole is said to possess the " $t-\phi$ orthogonality property" if the 2-planes spanned by $t^{a}$ and the rotational Killing field $\phi^{a}$ are orthogonal to a family of 2-dimensional surfaces. The $t-\phi$ orthogonality property holds for all stationary-axisymmetric black hole solutions to the vacuum Einstein or Einstein-Maxwell equations (see, e.g., [56]).

A null surface, $\mathcal{K}$, whose null generators coincide with the orbits of a oneparameter group of isometries (so that there is a Killing field $\xi^{a}$ normal to $\mathcal{K}$ ) is called a Killing horizon. There are two independent results (usually referred to as "rigidity theorems") that show that in a wide variety of cases of interest, the event horizon, $\mathcal{H}$, of a stationary black hole must be a Killing horizon. The first, due to Carter [35], states that for a static black hole, the static Killing field $t^{a}$ must be normal to the horizon, whereas for a stationary-axisymmetric black hole with the $t-\phi$ orthogonality property there exists a Killing field $\xi^{a}$ of the form

$$
\xi^{a}=t^{a}+\Omega \phi^{a}
$$

which is normal to the event horizon. The constant $\Omega$ defined by Eq. (2) is called the angular velocity of the horizon. Carter's result does not rely on any field equations, but leaves open the possibility that there could exist stationary black holes without the above symmetries whose event horizons are not Killing horizons. The second result, due to Hawking [5.5] (see also [45]), directly proves

Living Reviews in Relativity (2001-6)

http://www.livingreviews.org 
that in vacuum or electrovac general relativity, the event horizon of any stationary black hole must be a Killing horizon. Consequently, if $t^{a}$ fails to be normal to the horizon, then there must exist an additional Killing field, $\xi^{a}$, which is normal to the horizon, i.e., a stationary black hole must be nonrotating (from which staticity follows [84, 85., 37]) or axisymmetric (though not necessarily with the $t-\phi$ orthogonality property). Note that Hawking's theorem makes no assumptions of symmetries beyond stationarity, but it does rely on the properties of the field equations of general relativity.

Now, let $\mathcal{K}$ be any Killing horizon (not necessarily required to be the event horizon, $\mathcal{H}$, of a black hole), with normal Killing field $\xi^{a}$. Since $\nabla^{a}\left(\xi^{b} \xi_{b}\right)$ also is normal to $\mathcal{K}$, these vectors must be proportional at every point on $\mathcal{K}$. Hence, there exists a function, $\kappa$, on $\mathcal{K}$, known as the surface gravity of $\mathcal{K}$, which is defined by the equation

$$
\nabla^{a}\left(\xi^{b} \xi_{b}\right)=-2 \kappa \xi^{a} .
$$

It follows immediately that $\kappa$ must be constant along each null geodesic generator of $\mathcal{K}$, but, in general, $\kappa$ can vary from generator to generator. It is not difficult to show (see, e.g., [9.9]) that

$$
\kappa=\lim (V a),
$$

where $a$ is the magnitude of the acceleration of the orbits of $\xi^{a}$ in the region off of $\mathcal{K}$ where they are timelike, $V \equiv\left(-\xi^{a} \xi_{a}\right)^{1 / 2}$ is the "redshift factor" of $\xi^{a}$, and the limit as one approaches $\mathcal{K}$ is taken. Equation (四) motivates the terminology "surface gravity". Note that the surface gravity of a black hole is defined only when it is "in equilibrium", i.e., stationary, so that its event horizon is a Killing horizon. There is no notion of the surface gravity of a general, non-stationary black hole, although the definition of surface gravity can be extended to isolated horizons (see below).

In parallel with the two independent "rigidity theorems" mentioned above, there are two independent versions of the zeroth law of black hole mechanics. The first, due to Carter [35] (see also [78]), states that for any black hole which is static or is stationary-axisymmetric with the $t-\phi$ orthogonality property, the surface gravity $\kappa$, must be constant over its event horizon $\mathcal{H}$. This result is purely geometrical, i.e., it involves no use of any field equations. The second, due to Bardeen, Carter, and Hawking [II2] states that if Einstein's equation holds with the matter stress-energy tensor satisfying the dominant energy condition, then $\kappa$ must be constant on any Killing horizon. Thus, in the second version of the zeroth law, the hypothesis that the $t-\phi$ orthogonality property holds is eliminated, but use is made of the field equations of general relativity.

A bifurcate Killing horizon is a pair of null surfaces, $\mathcal{K}_{A}$ and $\mathcal{K}_{B}$, which intersect on a spacelike 2-surface, $\mathcal{C}$ (called the "bifurcation surface"), such that $\mathcal{K}_{A}$ and $\mathcal{K}_{B}$ are each Killing horizons with respect to the same Killing field $\xi^{a}$. It follows that $\xi^{a}$ must vanish on $\mathcal{C}$; conversely, if a Killing field, $\xi^{a}$, vanishes on a two-dimensional spacelike surface, $\mathcal{C}$, then $\mathcal{C}$ will be the bifurcation surface of a bifurcate Killing horizon associated with $\xi^{a}$ (see [IIU] for further discussion). An important consequence of the zeroth law is that if $\kappa \neq 0$, then in the "maximally

Living Reviews in Relativity (2001-6)

http://www.livingreviews.org 
extended" spacetime representing a stationary black hole, the event horizon, $\mathcal{H}$, comprises a branch of a bifurcate Killing horizon [[8]. This result is purely geometrical - involving no use of any field equations. As a consequence, the study of stationary black holes which satisfy the zeroth law divides into two cases: "extremal" black holes (for which, by definition, $\kappa=0$ ), and black holes with bifurcate horizons.

The first law of black hole mechanics is simply an identity relating the changes in mass, $M$, angular momentum, $J$, and horizon area, $A$, of a stationary black hole when it is perturbed. To first order, the variations of these quantities in the vacuum case always satisfy

$$
\delta M=\frac{1}{8 \pi} \kappa \delta A+\Omega \delta J .
$$

In the original derivation of this law [II2], it was required that the perturbation be stationary. Furthermore, the original derivation made use of the detailed form of Einstein's equation. Subsequently, the derivation has been generalized to hold for non-stationary perturbations [ $[\mathbf{8 4}, 60]$ ], provided that the change in area is evaluated at the bifurcation surface, $\mathcal{C}$, of the unperturbed black hole (see, however, $[\underline{80}]$ for a derivation of the first law for non-stationary perturbations that does not require evaluation at the bifurcation surface). More significantly, it has been shown $[\mathbf{6 0}]$ that the validity of this law depends only on very general properties of the field equations. Specifically, a version of this law holds for any field equations derived from a diffeomorphism covariant Lagrangian, $L$. Such a Lagrangian can always be written in the form

$$
L=L\left(g_{a b} ; R_{a b c d}, \nabla_{a} R_{b c d e}, \ldots ; \psi, \nabla_{a} \psi, \ldots\right),
$$

where $\nabla_{a}$ denotes the derivative operator associated with $g_{a b}, R_{a b c d}$ denotes the Riemann curvature tensor of $g_{a b}$, and $\psi$ denotes the collection of all matter fields of the theory (with indices suppressed). An arbitrary (but finite) number of derivatives of $R_{a b c d}$ and $\psi$ are permitted to appear in $L$. In this more general context, the first law of black hole mechanics is seen to be a direct consequence of an identity holding for the variation of the Noether current. The general form of the first law takes the form

$$
\delta M=\frac{\kappa}{2 \pi} \delta S_{\mathrm{bh}}+\Omega \delta J+\ldots,
$$

where the "..." denote possible additional contributions from long range matter fields, and where

$$
S_{\mathrm{bh}} \equiv-2 \pi \int_{\mathcal{C}} \frac{\delta L}{\delta R_{a b c d}} n_{a b} n_{c d}
$$

Here $n_{a b}$ is the binormal to the bifurcation surface $\mathcal{C}$ (normalized so that $n_{a b} n^{a b}=$ -2 ), and the functional derivative is taken by formally viewing the Riemann tensor as a field which is independent of the metric in Eq. (6). For the case of vacuum general relativity, where $L=R \sqrt{-g}$, a simple calculation yields

$$
S_{\mathrm{bh}}=A / 4
$$

Living Reviews in Relativity (2001-6)

http://www.livingreviews.org 
and Eq. (7) reduces to Eq. (5).

The close mathematical analogy of the zeroth, first, and second laws of thermodynamics to corresponding laws of classical black hole mechanics is broken by the Planck-Nernst form of the third law of thermodynamics, which states that $S \rightarrow 0$ (or a "universal constant") as $T \rightarrow 0$. The analog of this law fails in black hole mechanics - although analogs of alternative formulations of the third law do appear to hold for black holes [5.9] - since there exist extremal black holes (i.e., black holes with $\kappa=0$ ) with finite $A$. However, there is good reason to believe that the "Planck-Nernst theorem" should not be viewed as a fundamental law of thermodynamics [T] but rather as a property of the density of states near the ground state in the thermodynamic limit, which happens to be valid for commonly studied materials. Indeed, examples can be given of ordinary quantum systems that violate the Planck-Nernst form of the third law in a manner very similar to the violations of the analog of this law that occur for black holes [1102].

As discussed above, the zeroth and first laws of black hole mechanics have been formulated in the mathematical setting of stationary black holes whose event horizons are Killing horizons. The requirement of stationarity applies to the entire spacetime and, indeed, for the first law, stationarity of the entire spacetime is essential in order to relate variations of quantities defined at the horizon (like $A$ ) to variations of quantities defined at infinity (like $M$ and $J$ ). However, it would seem reasonable to expect that the equilibrium thermodynamic behavior of a black hole would require only a form of local stationarity at the event horizon. For the formulation of the first law of black hole mechanics, one would also then need local definitions of quantities like $M$ and $J$ at the horizon. Such an approach toward the formulation of the laws of black hole mechanics has recently been taken via the notion of an isolated horizon, defined as a null hypersurface with vanishing shear and expansion satisfying the additional properties stated in [4]. (This definition supersedes the more restrictive definitions given, e.g., in [5, 6, [7].) The presence of an isolated horizon does not require the entire spacetime to be stationary [65]. A direct analog of the zeroth law for stationary event horizons can be shown to hold for isolated horizons [ $[9]$. In the Einstein-Maxwell case, one can demand (via a choice of scaling of the normal to the isolated horizon as well as a choice of gauge for the Maxwell field) that the surface gravity and electrostatic potential of the isolated horizon be functions of only its area and charge. The requirement that time evolution be symplectic then leads to a version of the first law of black hole mechanics as well as a (in general, non-unique) local notion of the energy of the isolated horizon [9]. These results also have been generalized to allow dilaton couplings [7] and Yang-Mills fields [38, 9 ].

In comparing the laws of black hole mechanics in classical general relativity with the laws of thermodynamics, it should first be noted that the black hole uniqueness theorems (see, e.g., [56]) establish that stationary black holes - i.e., black holes "in equilibrium" - are characterized by a small number of parameters, analogous to the "state parameters" of ordinary thermodynamics. In the corresponding laws, the role of energy, $E$, is played by the mass, $M$, of

Living Reviews in Relativity (2001-6)

http://www.livingreviews.org 
the black hole; the role of temperature, $T$, is played by a constant times the surface gravity, $\kappa$, of the black hole; and the role of entropy, $S$, is played by a constant times the area, $A$, of the black hole. The fact that $E$ and $M$ represent the same physical quantity provides a strong hint that the mathematical analogy between the laws of black hole mechanics and the laws of thermodynamics might be of physical significance. However, as argued in [12], this cannot be the case in classical general relativity. The physical temperature of a black hole is absolute zero (see subsection 4.1 below), so there can be no physical relationship between $T$ and $\kappa$. Consequently, it also would be inconsistent to assume a physical relationship between $S$ and $A$. As we shall now see, this situation changes dramatically when quantum effects are taken into account.

Living Reviews in Relativity (2001-6)

http://www.livingreviews.org 


\section{Hawking Radiation}

In 1974, Hawking [54] made the startling discovery that the physical temperature of a black hole is not absolute zero: As a result of quantum particle creation effects, a black hole radiates to infinity all species of particles with a perfect black body spectrum, at temperature (in units with $G=c=\hbar=k=1$ )

$$
T=\frac{\kappa}{2 \pi} .
$$

Thus, $\kappa / 2 \pi$ truly is the physical temperature of a black hole, not merely a quantity playing a role mathematically analogous to temperature in the laws of black hole mechanics. In this section, we review the status of the derivation of the Hawking effect and also discuss the closely related Unruh effect.

The original derivation of the Hawking effect [54] made direct use of the formalism for calculating particle creation in a curved spacetime that had been developed by Parker [ [.3.] and others. Hawking considered a classical spacetime $\left(M, g_{a b}\right)$ describing gravitational collapse to a Schwarzschild black hole. He then considered a free (i.e., linear) quantum field propagating in this background spacetime, which is initially in its vacuum state prior to the collapse, and he computed the particle content of the field at infinity at late times. This calculation involves taking the positive frequency mode function corresponding to a particle state at late times, propagating it backwards in time, and determining its positive and negative frequency parts in the asymptotic past. His calculation revealed that at late times, the expected number of particles at infinity corresponds to emission from a perfect black body (of finite size) at the Hawking temperature (Eq. (10)). It should be noted that this result relies only on the analysis of quantum fields in the region exterior to the black hole, and it does not make use of any gravitational field equations.

The original Hawking calculation can be straightforwardly generalized and extended in the following ways. First, one may consider a spacetime representing an arbitrary gravitational collapse to a black hole such that the black hole "settles down" to a stationary final state satisfying the zeroth law of black hole mechanics (so that the surface gravity, $\kappa$, of the black hole final state is constant over its event horizon). The initial state of the quantum field may be taken to be any nonsingular state (i.e., any Hadamard state - see, e.g., [ㅍ]]) rather than the initial vacuum state. Finally, it can be shown [98] that all aspects of the final state at late times (i.e., not merely the expected number of particles in each mode) correspond to black body] thermal radiation emanating from the black hole at temperature (Eq. (10)).

It should be noted that no infinities arise in the calculation of the Hawking effect for a free field, so the results are mathematically well defined, without any need for regularization or renormalization. The original derivations [54, 98] made use of notions of "particles propagating into the black hole", but the results for what an observer sees at infinity were shown to be independent of

\footnotetext{
${ }^{1}$ If the black hole is rotating, the the spectrum seen by an observer at infinity corresponds to what would emerge from a "rotating black body".
}

Living Reviews in Relativity (2001-6)

http://www.livingreviews.org 
the ambiguities inherent in such notions and, indeed, a derivation of the Hawking effect has been given [44] which entirely avoids the introduction of any notion of "particles". However, there remains one significant difficultly with the Hawking derivation: In the calculation of the backward-in-time propagation of a mode, it is found that the mode undergoes a large blueshift as it propagates near the event horizon, but there is no correspondingly large redshift as the mode propagates back through the collapsing matter into the asymptotic past. Indeed, the net blueshift factor of the mode is proportional to $\exp (\kappa t)$, where $t$ is the time that the mode would reach an observer at infinity. Thus, within a time of order $1 / \kappa$ of the formation of a black hole (i.e., $\sim 10^{-5}$ seconds for a one solar mass Schwarzschild black hole), the Hawking derivation involves (in its intermediate steps) the propagation of modes of frequency much higher than the Planck frequency. In this regime, it is difficult to believe in the accuracy of free field theory - or any other theory known to mankind.

An approach to investigating this issue was first suggested by Unruh [92], who noted that a close analog of the Hawking effect occurs for quantized sound waves in a fluid undergoing supersonic flow. A similar blueshifting of the modes quickly brings one into a regime well outside the domain of validity of the continuum fluid equations. Unruh suggested replacing the continuum fluid equations with a more realistic model at high frequencies to see if the fluid analog of the Hawking effect would still occur. More recently, Unruh investigated models where the dispersion relation is altered at ultra-high frequencies, and he found no deviation from the Hawking prediction [93]. A variety of alternative models have been considered by other researchers [28, 3.9, 62, [7.9, 97, 40, 63]. Again, agreement with the Hawking effect prediction was found in all cases, despite significant modifications of the theory at high frequencies.

The robustness of the Hawking effect with respect to modifications of the theory at ultra-high frequency probably can be understood on the following grounds. One may view the backward-in-time propagation of modes as consisting of two stages: a first stage where the blueshifting of the mode brings it into a WKB regime but the frequencies remain well below the Planck scale, and a second stage where the continued blueshifting takes one to the Planck scale and beyond. In the first stage, the usual field theory calculations should be reliable. On the other hand, after the mode has entered a WKB regime, it seems plausible that the kinds of modifications to its propagation laws considered in [93, [28, [3.9, 62, [7.9, 97, 40, 63] should not affect its essential properties, in particular the magnitude of its negative frequency part.

Indeed, an issue closely related to the validity of the original Hawking derivation arises if one asks how a uniformly accelerating observer in Minkowski spacetime perceives the ordinary (inertial) vacuum state (see below). The outgoing modes of a given frequency $\omega$ as seen by the accelerating observer at proper time $\tau$ along his worldline correspond to modes of frequency $\sim \omega \exp (a \tau)$ in a fixed inertial frame. Therefore, at time $\tau \gg 1 / a$ one might worry about fieldtheoretic derivations of what the accelerating observer would see. However, in this case one can appeal to Lorentz invariance to argue that what the accelerating observer sees cannot change with time. It seems likely that one could

Living Reviews in Relativity (2001-6)

http://www.livingreviews.org 
similarly argue that the Hawking effect cannot be altered by modifications of the theory at ultra-high frequencies, provided that these modifications preserve an appropriate "local Lorentz invariance" of the theory. Thus, there appears to be strong reasons for believing in the validity of the Hawking effect despite the occurrence of ultra-high-frequency modes in the derivation.

There is a second, logically independent result - namely, the Unruh effect [91] and its generalization to curved spacetime - which also gives rise to the formula (10). Although the Unruh effect is mathematically very closely related to the Hawking effect, it is important to distinguish clearly between them. In its most general form, the Unruh effect may be stated as follows (see [64, ए0I] for further discussion): Consider a classical spacetime $\left(M, g_{a b}\right)$ that contains a bifurcate Killing horizon, $\mathcal{K}=\mathcal{K}_{A} \cup \mathcal{K}_{B}$, so that there is a one-parameter group of isometries whose associated Killing field, $\xi^{a}$, is normal to $\mathcal{K}$. Consider a free quantum field on this spacetime. Then there exists at most one globally nonsingular state of the field which is invariant under the isometries. Furthermore, in the "wedges" of the spacetime where the isometries have timelike orbits, this state (if it exists) is a KMS (i.e., thermal equilibrium) state at temperature (10) with respect to the isometries.

Note that in Minkowski spacetime, any one-parameter group of Lorentz boosts has an associated bifurcate Killing horizon, comprised by two intersecting null planes. The unique, globally nonsingular state which is invariant under these isometries is simply the usual ("inertial") vacuum state, $|0\rangle$. In the "right and left wedges" of Minkowski spacetime defined by the Killing horizon, the orbits of the Lorentz boost isometries are timelike, and, indeed, these orbits correspond to worldlines of uniformly accelerating observers. If we normalize the boost Killing field, $b^{a}$, so that Killing time equals proper time on an orbit with acceleration $a$, then the surface gravity of the Killing horizon is $\kappa=a$. An observer following this orbit would naturally use $b^{a}$ to define a notion of "time translation symmetry". Consequently, by the above general result, when the field is in the inertial vacuum state, a uniformly accelerating observer would describe the field as being in a thermal equilibrium state at temperature

$$
T=\frac{a}{2 \pi}
$$

as originally discovered by Unruh [9]]. A mathematically rigorous proof of the Unruh effect in Minkowski spacetime was given by Bisognano and Wichmann [2:3] in work motivated by entirely different considerations (and done independently of and nearly simultaneously with the work of Unruh). Furthermore, the Bisognano-Wichmann theorem is formulated in the general context of axiomatic quantum field theory, thus establishing that the Unruh effect is not limited to free field theory.

Although there is a close mathematical relationship between the Unruh effect and the Hawking effect, it should be emphasized that these results refer to different states of the quantum field. We can divide the late time modes of the quantum field in the following manner, according to the properties that they would have in the analytically continued spacetime [[8] representing the asymp-

Living Reviews in Relativity (2001-6)

http://www.livingreviews.org 
totic final stationary state of the black hole: We refer to modes that would have emanated from the white hole region of the analytically continued spacetime as "UP modes" and those that would have originated from infinity as "IN modes". In the Hawking effect, the asymptotic final state of the quantum field is a state in which the UP modes of the quantum field are thermally populated at temperature (10), but the IN modes are unpopulated. This state (usually referred to as the "Unruh vacuum") would be singular on the white hole horizon in the analytically continued spacetime. On the other hand, in the Unruh effect and its generalization to curved spacetimes, the state in question (usually referred to as the "Hartle-Hawking vacuum" [52]) is globally nonsingular, and all modes of the quantum field in the "left and right wedges" are thermally populated.2]

The differences between the Unruh and Hawking effects can be seen dramatically in the case of a Kerr black hole. For the Kerr black hole, it can be shown [64] that there does not exist any globally nonsingular state of the field which is invariant under the isometries associated with the Killing horizon, i.e., there does not exist a "Hartle-Hawking vacuum state" on Kerr spacetime. However, there is no difficultly with the derivation of the Hawking effect for Kerr black holes, i.e., the "Unruh vacuum state" does exist.

It should be emphasized that in the Hawking effect, the temperature (10) represents the temperature as measured by an observer near infinity. For any observer following an orbit of the Killing field, $\xi^{a}$, normal to the horizon, the locally measured temperature of the UP modes is given by

$$
T=\frac{\kappa}{2 \pi V},
$$

where $V=\left(-\xi^{a} \xi_{a}\right)^{1 / 2}$. In other words, the locally measured temperature of the Hawking radiation follows the Tolman law. Now, as one approaches the horizon of the black hole, the UP modes dominate over the IN modes. Taking Eq. (4) into account, we see that $T \rightarrow a / 2 \pi$ as the black hole horizon, $\mathcal{H}$, is approached, i.e., in this limit Eq. (12) corresponds to the flat spacetime Unruh effect.

Equation (12) shows that when quantum effects are taken into account, a black hole is surrounded by a "thermal atmosphere" whose local temperature as measured by observers following orbits of $\xi^{a}$ becomes divergent as one approaches the horizon. As we shall see in the next section, this thermal atmosphere produces important physical effects on quasi-stationary bodies near the black hole. On the other hand, it should be emphasized that for a macroscopic black hole, observers who freely fall into the black hole would not notice any important quantum effects as they approach and cross the horizon.

\footnotetext{
${ }^{2}$ The state in which none of the modes in the region exterior to the black hole are populated is usually referred to as the "Boulware vacuum". The Boulware vacuum is singular on both the black hole and white hole horizons.
}

Living Reviews in Relativity (2001-6) http://www.livingreviews.org 


\section{The Generalized Second Law (GSL)}

In this section, we shall review some arguments for the validity of the generalized second law (GSL). We also shall review the status of several proposed entropy bounds on matter that have played a role in discussions and analyses of the GSL.

\subsection{Arguments for the validity of the GSL}

Even in classical general relativity, there is a serious difficulty with the ordinary second law of thermodynamics when a black hole is present, as originally emphasized by J.A. Wheeler: One can simply take some ordinary matter and drop it into a black hole, where, according to classical general relativity, it will disappear into a spacetime singularity. In this process, one loses the entropy initially present in the matter, and no compensating gain of ordinary entropy occurs, so the total entropy, $S$, of matter in the universe decreases. One could attempt to salvage the ordinary second law by invoking the bookkeeping rule that one must continue to count the entropy of matter dropped into a black hole as still contributing to the total entropy of the universe. However, the second law would then have the status of being observationally unverifiable.

As already mentioned in section 2, after the area theorem was proven, Bekenstein [14, 15] proposed a way out of this difficulty: Assign an entropy, $S_{\mathrm{bh}}$, to a black hole given by a numerical factor of order unity times the area, $A$, of the black hole in Planck units. Define the generalized entropy, $S^{\prime}$, to be the sum of the ordinary entropy, $S$, of matter outside of a black hole plus the black hole entropy

$$
S^{\prime} \equiv S+S_{\mathrm{bh}} .
$$

Finally, replace the ordinary second law of thermodynamics by the generalized second law (GSL): The total generalized entropy of the universe never decreases with time,

$$
\Delta S^{\prime} \geq 0 .
$$

Although the ordinary second law will fail when matter is dropped into a black hole, such a process will tend to increase the area of the black hole, so there is a possibility that the GSL will hold.

Bekenstein's proposal of the GSL was made prior to the discovery of Hawking radiation. When Hawking radiation is taken into account, a serious problem also arises with the second law of black hole mechanics (i.e., the area theorem): Conservation of energy requires that an isolated black hole must lose mass in order to compensate for the energy radiated to infinity by the Hawking process. Indeed, if one equates the rate of mass loss of the black hole to the energy flux at infinity due to particle creation, one arrives at the startling conclusion that an isolated black hole will radiate away all of its mass within a finite time. During this process of black hole "evaporation", $A$ will decrease. Such an area decrease can occur because the expected stress-energy tensor of quantum matter does

Living Reviews in Relativity (2001-6)

http://www.livingreviews.org 
not satisfy the null energy condition - even for matter for which this condition holds classically - in violation of a key hypothesis of the area theorem.

However, although the second law of black hole mechanics fails during the black hole evaporation process, if we adjust the numerical factor in the definition of $S_{\mathrm{bh}}$ to correspond to the identification of $\kappa / 2 \pi$ as temperature in the first law of black hole mechanics - so that, as in Eq. (9) above, we have $S_{\mathrm{bh}}=A / 4$ in Planck units - then the GSL continues to hold: Although $A$ decreases, there is at least as much ordinary entropy generated outside the black hole by the Hawking process. Thus, although the ordinary second law fails in the presence of black holes and the second law of black hole mechanics fails when quantum effects are taken into account, there is a possibility that the GSL may always hold. If the GSL does hold, it seems clear that we must interpret $S_{\mathrm{bh}}$ as representing the physical entropy of a black hole, and that the laws of black hole mechanics must truly represent the ordinary laws of thermodynamics as applied to black holes. Thus, a central issue in black hole thermodynamics is whether the GSL holds in all processes.

It was immediately recognized by Bekenstein [14] (see also [12]) that there is a serious difficulty with the GSL if one considers a process wherein one carefully lowers a box containing matter with entropy $S$ and energy $E$ very close to the horizon of a black hole before dropping it in. Classically, if one could lower the box arbitrarily close to the horizon before dropping it in, one would recover all of the energy originally in the box as "work" at infinity. No energy would be delivered to the black hole, so by the first law of black hole mechanics, Eq. (7), the black hole area, $A$, would not increase. However, one would still get rid of all of the entropy, $S$, originally in the box, in violation of the GSL.

Indeed, this process makes manifest the fact that in classical general relativity, the physical temperature of a black hole is absolute zero: The above process is, in effect, a Carnot cycle which converts "heat" into "work" with $100 \%$ efficiency [4.9]. The difficulty with the GSL in the above process can be viewed as stemming from an inconsistency of this fact with the mathematical assignment of a finite (non-zero) temperature to the black hole required by the first law of black hole mechanics if one assigns a finite (non-infinite) entropy to the black hole.

Bekenstein proposed a resolution of the above difficulty with the GSL in a quasi-static lowering process by arguing [14, [15] that it would not be possible to lower a box containing physically reasonable matter close enough to the horizon of the black hole to violate the GSL. As will be discussed further in the next sub-section, this proposed resolution was later refined by postulating a universal bound on the entropy of systems with a given energy and size [16]. However, an alternate resolution was proposed in [94], based upon the idea that, when quantum effects are taken into account, the physical temperature of a black hole is no longer absolute zero, but rather is the Hawking temperature, $\kappa / 2 \pi$. Since the Hawking temperature goes to zero in the limit of a large black hole, it might appear that quantum effects could not be of much relevance in this case. However, despite the fact that Hawking radiation at infinity is indeed negligible for large black holes, the effects of the quantum "thermal

Living Reviews in Relativity (2001-6)

http://www.livingreviews.org 
atmosphere" surrounding the black hole are not negligible on bodies that are quasi-statically lowered toward the black hole. The temperature gradient in the thermal atmosphere (see Eq. (12)) implies that there is a pressure gradient and, consequently, a buoyancy force on the box. This buoyancy force becomes infinitely large in the limit as the box is lowered to the horizon. As a result of this buoyancy force, the optimal place to drop the box into the black hole is no longer the horizon but rather the "floating point" of the box, where its weight is equal to the weight of the displaced thermal atmosphere. The minimum area increase given to the black hole in the process is no longer zero, but rather turns out to be an amount just sufficient to prevent any violation of the GSL from occurring in this process [94].

The analysis of [94] considered only a particular class of gedankenexperiments for violating the GSL involving the quasi-static lowering of a box near a black hole. Of course, since one does not have a general proof of the ordinary second law of thermodynamics - and, indeed, for finite systems, there should always be a nonvanishing probability of violating the ordinary second law - it would not be reasonable to expect to obtain a completely general proof of the GSL. However, general arguments within the semiclassical approximation for the validity of the GSL for arbitrary infinitesimal quasi-static processes have been given in [105, 90, 101]. These arguments crucially rely on the presence of the thermal atmosphere surrounding the black hole. Related arguments for the validity of the GSL have been given in [48, 82]. In [48], it is assumed that the incoming state is a product state of radiation originating from infinity (i.e., IN modes) and radiation that would appear to emanate from the white hole region of the analytically continued spacetime (i.e., UP modes), and it is argued that the generalized entropy must increase under unitary evolution. In [ [82], it is argued on quite general grounds that the (generalized) entropy of the state of the region exterior to the black hole must increase under the assumption that it undergoes autonomous evolution.

Indeed, it should be noted that if one could violate the GSL for an infinitesimal quasi-static process in a regime where the black hole can be treated semi-classically, then it also should be possible to violate the ordinary second law for a corresponding process involving a self-gravitating body. Namely, suppose that the GSL could be violated for an infinitesimal quasi-static process involving, say, a Schwarzschild black hole of mass $M$ (with $M$ much larger than the Planck mass). This process might involve lowering matter towards the black hole and possibly dropping the matter into it. However, an observer doing this lowering or dropping can "probe" only the region outside of the black hole, so there will be some $r_{0}>2 M$ such that the detailed structure of the black hole will directly enter the analysis of the process only for $r>r_{0}$. Now replace the black hole by a shell of matter of mass $M$ and radius $r_{0}$, and surround this shell with a "real" atmosphere of radiation in thermal equilibrium at the Hawking temperature (10) as measured by an observer at infinity. Then the ordinary second law should be violated when one performs the same process to the shell surrounded by the ("real") thermal atmosphere as one performs to violate the GSL when the black hole is present. Indeed, the arguments of [105, 90, 미]

Living Reviews in Relativity (2001-6)

http://www. livingreviews.org 
do not distinguish between infinitesimal quasi-static processes involving a black hole as compared with a shell surrounded by a ("real") thermal atmosphere at the Hawking temperature.

In summary, there appear to be strong grounds for believing in the validity of the GSL.

\subsection{Entropy bounds}

As discussed in the previous subsection, for a classical black hole the GSL would be violated if one could lower a box containing matter sufficiently close to the black hole before dropping it in. Indeed, for a Schwarzschild black hole, a simple calculation reveals that if the size of the box can be neglected, then the GSL would be violated if one lowered a box containing energy $E$ and entropy $S$ to within a proper distance $D$ of the bifurcation surface of the event horizon before dropping it in, where

$$
D<\frac{S}{(2 \pi E)} .
$$

(This formula holds independently of the mass, $M$, of the black hole.) However, it is far from clear that the finite size of the box can be neglected if one lowers a box containing physically reasonable matter this close to the black hole. If it cannot be neglected, then this proposed counterexample to the GSL would be invalidated.

As already mentioned in the previous subsection, these considerations led Bekenstein [16] to propose a universal bound on the entropy-to-energy ratio of bounded matter, given by

$$
S / E \leq 2 \pi R,
$$

where $R$ denotes the "circumscribing radius" of the body. Here " $E$ " is normally interpreted as the energy above the ground state; otherwise, Eq. (16) would be trivially violated in cases where the Casimir energy is negative [ri0] - although in such cases in may still be possible to rescue Eq. (16) by postulating a suitable minimum energy of the box walls [[13].

Two key questions one can ask about this bound are: (1) Does it hold in nature? (2) Is it needed for the validity of the GSL? With regard to question (1), even in Minkowski spacetime, there exist many model systems that are physically reasonable (in the sense of positive energies, causal equations of state, etc.) for which Eq. (16) fails. (For a recent discussion of such counterexamples

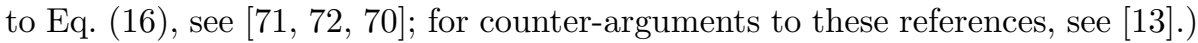
In particular it is easily seen that for a system consisting of $N$ non-interacting species of particles with identical properties, Eq. (16) must fail when $N$ becomes sufficiently large. However, for a system of $N$ species of free, massless bosons or fermions, one must take $N$ to be enormously large [18] to violate Eq. (16]), so it does not appear that nature has chosen to take advantage of this possible means of violating (16). Equation (16) also is violated at sufficiently low temperatures if one defines the entropy, $S$, of the system via the canonical ensemble, i.e., $S(T)=-\operatorname{tr}[\rho \ln \rho]$, where $\rho$ denotes the canonical ensemble density matrix,

Living Reviews in Relativity (2001-6)

http://www.livingreviews.org 


$$
\rho=\exp (-H / T) \operatorname{tr}[\exp (-H / T)],
$$

where $H$ is the Hamiltonian. However, a study of a variety of model systems [18] indicates that (16) holds at low temperatures when $S$ is defined via the microcanonical ensemble, i.e., $S(E)=\ln n$ where $n$ is the density of quantum states with energy $E$. More generally, Eq. (16) has been shown to hold for a wide variety of systems in flat spacetime [18, [22].

The status of Eq. (16) in curved spacetime is unclear; indeed, while there is some ambiguity in how " $E$ " and " $R$ " are defined in Minkowski spacetime [70], it is very unclear what these quantities would mean in a general, non-sphericallysymmetric spacetime. (These same difficulties also plague attempts to give a mathematically rigorous formulation of the "hoop conjecture" [68].) With regard to " $E$ ", it has long been recognized that there is no meaningful local notion of gravitational energy density in general relativity. Although numerous proposals have been made to define a notion of "quasi-local mass" associated with a closed 2-surface (see, e.g., [ [77, 30] ), none appear to have fully satisfactory properties. Although the difficulties with defining a localized notion of energy are well known, it does not seem to be as widely recognized that there also are serious difficulties in defining " $R$ ": Given any spacelike 2 -surface, $\mathcal{C}$, in a 4-dimensional spacetime and given any open neighborhood, $\mathcal{O}$, of $\mathcal{C}$, there exists a spacelike 2 -surface, $\mathcal{C}^{\prime}$ (composed of nearly null portions) contained within $\mathcal{O}$ with arbitrarily small area and circumscribing radius. Thus, if one is given a system confined to a world tube in spacetime, it is far from clear how to define any notion of the "externally measured size" of the region unless, say, one is given a preferred slicing by spacelike hypersurfaces. Nevertheless, the fact that Eq. (16) holds for the known black hole solutions (and, indeed, is saturated by the Schwarzschild black hole) and also plausibly holds for a self-gravitating spherically symmetric body [8.3] provides an indication that some version of (16) may hold in curved spacetime.

With regard to question (2), in the previous section we reviewed arguments for the validity of the GSL that did not require the invocation of any entropy bounds. Thus, the answer to question (2) is "no" unless there are deficiencies in the arguments of the previous section that invalidate their conclusions. A number of such potential deficiencies have been pointed out by Bekenstein. Specifically, the analysis and conclusions of [94] have been criticized by Bekenstein on the grounds that:

i. A "thin box" approximation was made [I7]

ii. It is possible to have a box whose contents have a greater entropy than unconfined thermal radiation of the same energy and volume [T7].

iii. Under certain assumptions concerning the size/shape of the box, the nature of the thermal atmosphere, and the location of the floating point, the buoyancy force of the thermal atmosphere can be shown to be negligible and thus cannot play a role in enforcing the GSL [IIT].

Living Reviews in Relativity (2001-6)

http://www.livingreviews.org 
iv. Under certain other assumptions, the box size at the floating point will be smaller than the typical wavelengths in the ambient thermal atmosphere, thus likely decreasing the magnitude of the buoyancy force [2]].

Responses to criticism (i) were given in [95] and [75]; a response to criticism (ii) was given in [9.5]; and a response to (iii) was given in [75]. As far as I am a aware, no response to (iv) has yet been given in the literature except to note [43] that the arguments of [2I] should pose similar difficulties for the ordinary second law for gedankenexperiments involving a self-gravitating body (see the end of subsection 4.1 above). Thus, my own view is that Eq. (16) is not necessary for the validity of the GSL 3 . However, this conclusion remains controversial; see [ [2] for a recent discussion.

More recently, an alternative entropy bound has been proposed: It has been suggested that the entropy contained within a region whose boundary has area $A$ must satisfy [8.9, [201, 86] $]$

$$
S \leq A / 4 .
$$

This proposal is closely related to the "holographic principle", which, roughly speaking, states that the physics in any spatial region can be fully described in terms of the degrees of freedom associated with the boundary of that region. (The literature on the holographic principle is far too extensive and rapidly developing to attempt to give any review of it here.) The bound (18) would follow from (16) under the additional assumption of small self-gravitation (so that $E \lesssim R$ ). Thus, many of the arguments in favor of (16) are also applicable to (18). Similarly, the counterexample to (16) obtained by taking the number, $N$, of particle species sufficiently large also provides a counterexample to (18), so it appears that (18) can, in principle, be violated by physically reasonable systems (although not necessarily by any systems actually occurring in nature).

Unlike Eq. (16), the bound (18) explicitly involves the gravitational constant $G$ (although we have set $G=1$ in all of our formulas), so there is no flat spacetime version of (18) applicable when gravity is "turned off". Also unlike (16), the bound (18) does not make reference to the energy, $E$, contained within the region, so the difficulty in defining $E$ in curved spacetime does not affect the formulation of (18). However, the above difficulty in defining the "bounding area", $A$, of a world tube in a general, curved spacetime remains present (but see below).

The following argument has been given that the bound (18) is necessary for the validity of the GSL [86]: Suppose we had a spherically symmetric system that was not a black hole (so $R>2 E$ ) and which violated the bound (18), so that $S>A / 4=\pi R^{2}$. Now collapse a spherical shell of mass $M=R / 2-E$ onto the system. A Schwarzschild black hole of radius $R$ should result. But the

\footnotetext{
${ }^{3}$ It is worth noting that if the buoyancy effects of the thermal atmosphere were negligible, the bound (16) also would not be sufficient to ensure the validity of the GSL for non-spherical bodies: The bound (16) is formulated in terms of the "circumscribing radius", i.e., the largest linear dimension, whereas if buoyancy effects were negligible, then to enforce the GSL one would need a bound of the form (16) with $R$ being the smallest linear dimension.
}

Living Reviews in Relativity (2001-6) http://www.livingreviews.org 
entropy of such a black hole is $A / 4$, so the generalized entropy will decrease in this process.

I am not aware of any counter-argument in the literature to the argument given in the previous paragraph, so I will take the opportunity to give one here. If there were a system which violated the bound (18), then the above argument shows that it would be (generalized) entropically unfavorable to collapse that system to a black hole. I believe that the conclusion one should draw from this is that, in this circumstance, it should not be possible to form a black hole. In other words, the bound (18) should be necessary in order for black holes to be stable or metastable states, but should not be needed for the validity of the GSL.

This viewpoint is supported by a simple model calculation. Consider a massless gas composed of $N$ species of (boson or fermion) particles confined by a spherical box of radius $R$. Then (neglecting self-gravitational effects and any corrections due to discreteness of modes) we have

$$
S \sim N^{1 / 4} R^{3 / 4} E^{3 / 4} .
$$

We wish to consider a configuration that is not already a black hole, so we need $E<R / 2$. To violate (18) - and thereby threaten to violate the GSL by collapsing a shell upon the system - we need to have $S>\pi R^{2}$. This means that we need to consider a model with $N \gtrsim R^{2}$. For such a model, start with a region $R$ containing matter with $S>\pi R^{2}$ but with $E<R / 2$. If we try to collapse a shell upon the system to form a black hole of radius $R$, the collapse time will be $\gtrsim R$. But the Hawking evaporation timescale in this model is $t_{\mathrm{H}} \sim R^{3} / N$, since the flux of Hawking radiation is proportional to $N$. Since $N \gtrsim R^{2}$, we have $t_{\mathrm{H}} \lesssim R$, so the Hawking evaporation time is shorter than the collapse time! Consequently, the black hole will never actually form. Rather, at best it will merely act as a catalyst for converting the original high entropy confined state into an even higher entropy state of unconfined Hawking radiation.

As mentioned above, the proposed bound (18) is ill defined in a general (non-spherically-symmetric) curved spacetime. There also are other difficulties with (18): In a closed universe, it is not obvious what constitutes the "inside" versus the "outside" of the bounding area. In addition, (18) can be violated near cosmological and other singularities, where the entropy of suitably chosen comoving volumes remains bounded away from zero but the area of the boundary of the region goes to zero. However, a reformulation of (18) which is well defined in a general curved spacetime and which avoids these difficulties has been given by Bousso [25, 26, 27]. Bousso's reformulation can be stated as follows: Let $\mathcal{L}$ be a null hypersurface such that the expansion, $\theta$, of $\mathcal{L}$ is everywhere non-positive, $\theta \leq 0$ (or, alternatively, is everywhere non-negative, $\theta \geq 0$ ). In particular, $\mathcal{L}$ is not allowed to contain caustics, where $\theta$ changes sign from $-\infty$ to $+\infty$. Let $B$ be a spacelike cross-section of $\mathcal{L}$. Bousso's reformulation conjectures that

$$
S_{\mathcal{L}} \leq A_{B} / 4
$$

where $A_{B}$ denotes the area of $B$ and $S_{\mathcal{L}}$ denotes the entropy flux through $\mathcal{L}$ to the future (or, respectively, the past) of $B$.

Living Reviews in Relativity (2001-6)

http://www.livingreviews.org 
In [43] it was argued that the bound (21) should be valid in certain "classical regimes" (see [43]) wherein the local entropy density of matter is bounded in a suitable manner by the energy density of matter. Furthermore, the following generalization of Bousso's bound was proposed: Let $\mathcal{L}$ be a null hypersurface which starts at a cross-section, $B$, and terminates at a cross-section $B^{\prime}$. Suppose further that $\mathcal{L}$ is such that its expansion, $\theta$, is either everywhere non-negative or everywhere non-positive. Then

$$
S_{\mathcal{L}} \leq\left|A_{B}-A_{B^{\prime}}\right| / 4 .
$$

Although we have argued above that the validity of the GSL should not depend upon the validity of the entropy bounds (16) or (18), there is a close relationship between the GSL and the generalized Bousso bound (21). Namely, as discussed in section 2 above, classically, the event horizon of a black hole is a null hypersurface satisfying $\theta \geq 0$. Thus, in a classical regime, the GSL itself would correspond to a special case of the generalized Bousso bound (21). This suggests the intriguing possibility that, in quantum gravity, there might be a more general formulation of the GSL - perhaps applicable to an arbitrary horizon as defined on p. 134 of [10T], not merely to an event horizon of a black hole - which would reduce to (21) in a suitable classical limit.

Living Reviews in Relativity (2001-6)

http://www.livingreviews.org 


\section{Calculations of Black Hole Entropy}

The considerations of the previous sections make a compelling case for the merger of the laws of black hole mechanics with the laws of thermodynamics. In particular, they strongly suggest that $S_{\mathrm{bh}}(=A / 4$ in general relativity see Eqs.(8) and (9) above) truly represents the physical entropy of a black hole. Now, the entropy of ordinary matter is understood to arise from the number of quantum states accessible to the matter at given values of the energy and other state parameters. One would like to obtain a similar understanding of why $A / 4$ represents the entropy of a black hole in general relativity by identifying (and counting) the quantum dynamical degrees of freedom of a black hole. In order to do so, it clearly will be necessary to go beyond the classical and semiclassical considerations of the previous sections and consider black holes within a fully quantum theory of gravity. In this section, we will briefly summarize some of the main approaches that have been taken to the direct calculation of the entropy of a black hole.

The first direct quantum calculation of black hole entropy was given by Gibbons and Hawking [50] in the context of Euclidean quantum gravity. They started with a formal, functional integral expression for the canonical ensemble partition function in Euclidean quantum gravity and evaluated it for a black hole in the "zero loop" (i.e, classical) approximation. As shown in [I01]], the mathematical steps in this procedure are in direct correspondence with the purely classical determination of the entropy from the form of the first law of black hole mechanics. A number of other entropy calculations that have been given within the formal framework of Euclidean quantum gravity also can be shown to be equivalent to the classical derivation (see [6I] for further discussion). Thus, although the derivation of [50] and other related derivations give some intriguing glimpses into possible deep relationships between black hole thermodynamics and Euclidean quantum gravity, they do not appear to provide any more insight than the classical derivation into accounting for the quantum degrees of freedom that are responsible for black hole entropy.

It should be noted that there is actually an inconsistency in the use of the canonical ensemble to derive a formula for black hole entropy, since the entropy of a black hole grows too rapidly with energy for the canonical ensemble to be defined. (Equivalently, the heat capacity of a Schwarzschild black hole is negative, so it cannot come to equilibrium with an infinite heat bath.) A derivation of black hole entropy using the microcanonical ensemble has been given in [2.9].

Another approach to the calculation of black hole entropy has been to attribute it to the "entanglement entropy" resulting from quantum field correlations between the exterior and interior of the black hole [24, 31, 57]. As a result of these correlations across the event horizon, the state of a quantum field when restricted to the exterior of the black hole is mixed. Indeed, in the absence of a short distance cutoff, the von Neumann entropy, $-\operatorname{tr}[\rho \ln \rho]$, of any physically reasonable state would diverge. If one now inserts a short distance cutoff of the order of the Planck scale, one obtains a von Neumann entropy of

Living Reviews in Relativity (2001-6)

http://www. livingreviews.org 
the order of the horizon area, $A$. Thus, this approach provides a natural way of accounting for why the entropy of a black hole is proportional to its surface area. However, the constant of proportionality depends upon a cutoff and is not (presently) calculable within this approach. (Indeed, one might argue that in this approach, the constant of proportionality between $S_{\mathrm{bh}}$ and $A$ should depend upon the number, $N$, of species of particles, and thus could not equal $1 / 4$ (independently of $N$ ). However, it is possible that the $N$-dependence in the number of states is compensated by an $N$-dependent renormalization of $G$ [ [87] and, hence, of the Planck scale cutoff.) More generally, it is far from clear why the black hole horizon should be singled out for a such special treatment of the quantum degrees of freedom in its vicinity, since similar quantum field correlations will exist across any other null surface. It is particularly puzzling why the local degrees of freedom associated with the horizon should be singled out since, as already noted in section 2 above, the black hole horizon at a given time is defined in terms of the entire future history of the spacetime and thus has no distinguished local significance. Finally, since the gravitational action and field equations play no role in the above derivation, it is difficult to see how this approach could give rise to a black hole entropy proportional to Eq. (8) (rather than proportional to $A$ ) in a more general theory of gravity. Similar remarks apply to approaches which attribute the relevant degrees of freedom to the "shape" of the horizon [ [8T] or to causal links crossing the horizon [4I].

A closely related idea has been to attribute the entropy of the black hole to the ordinary entropy of its thermal atmosphere $[\underline{\underline{88}}])$. If we assume that the thermal atmosphere behaves like a free, massless (boson or fermion) gas, its entropy density will be (roughly) proportional to $T^{3}$. However, since $T$ diverges near the horizon in the manner specified by Eq. (12), we find that the total entropy of the thermal atmosphere near the horizon diverges. This is, in effect, a new type of ultraviolet catastrophe. It arises because, on account of arbitrarily large redshifts, there now are infinitely many modes - of arbitrarily high locally measured frequency - that contribute a bounded energy as measured at infinity. To cure this divergence, it is necessary to impose a cutoff on the locally measured frequency of the modes. If we impose a cutoff of the order of the Planck scale, then the thermal atmosphere contributes an entropy of order the horizon area, $A$, just as in the entanglement entropy analysis. Indeed, this calculation is really the same as the entanglement entropy calculation, since the state of a quantum field outside of the black hole is thermal, so its von Neumann entropy is equal to its thermodynamic entropy (see also [6.9]). Note that the bulk of the entropy of the thermal atmosphere is highly localized in a "skin" surrounding the horizon, whose thickness is of order of the Planck length.

Since the attribution of black hole entropy to its thermal atmosphere is essentially equivalent to the entanglement entropy proposal, this approach has essentially the same strengths and weaknesses as the entanglement entropy approach. On one hand, it naturally accounts for a black hole entropy proportional to $A$. On the other hand, this result depends in an essential way on an uncalculable cutoff, and it is difficult to see how the analysis could give rise to Eq. (8) in a more general theory of gravity. The preferred status of the event horizon

Living Reviews in Relativity (2001-6) http://www.livingreviews.org 
and the localization of the degrees of freedom responsible for black hole entropy to a "Planck length skin" surrounding the horizon also remain puzzling in this approach. To see this more graphically, consider the collapse of a massive spherical shell of matter. Then, as the shell crosses its Schwarzschild radius, the spacetime curvature outside of the shell is still negligibly small. Nevertheless, within a time of order the Planck time after the crossing of the Schwarzschild radius, the "skin" of thermal atmosphere surrounding the newly formed black hole will come to equilibrium with respect to the notion of time translation symmetry for the static Schwarzschild exterior. Thus, if an entropy is to be assigned to the thermal atmosphere in the manner suggested by this proposal, then the degrees of freedom of the thermal atmosphere - which previously were viewed as irrelevant vacuum fluctuations making no contribution to entropy - suddenly become "activated" by the passage of the shell for the purpose of counting their entropy. A momentous change in the entropy of matter in the universe has occurred, even though observers riding on or near the shell see nothing of significance occurring.

Another approach that is closely related to the entanglement entropy and thermal atmosphere approaches - and which also contains elements closely related to the Euclidean approach and the classical derivation of Eq. (8) - attempts to account for black hole entropy in the context of Sakharov's theory of induced gravity [47, 46]. In Sakharov's proposal, the dynamical aspects of gravity arise from the collective excitations of massive fields. Constraints are then placed on these massive fields to cancel divergences and ensure that the effective cosmological constant vanishes. Sakharov's proposal is not expected to provide a fundamental description of quantum gravity, but at scales below the Planck scale it may possess features in common with other more fundamental descriptions. In common with the entanglement entropy and thermal atmosphere approaches, black hole entropy is explained as arising from the quantum field degrees of freedom outside the black hole. However, in this case the formula for black hole entropy involves a subtraction of the (divergent) mode counting expression and an (equally divergent) expression for the Noether charge operator, so that, in effect, only the massive fields contribute to black hole entropy. The result of this subtraction yields Eq. (9)).

More recently, another approach to the calculation of black hole entropy has been developed in the framework of quantum geometry [3, ए10]. In this approach, if one considers a spacetime containing an isolated horizon (see section 2 above), the classical symplectic form and classical Hamiltonian each acquire an additional boundary term arising from the isolated horizon [y]. (It should be noted that the phase space [ 8$]$ considered here incorporates the isolated horizon boundary conditions, i.e., only field variations that preserve the isolated horizon structure are admitted.) These additional terms are identical in form to that of a Chern-Simons theory defined on the isolated horizon. Classically, the fields on the isolated horizon are determined by continuity from the fields in the "bulk" and do not represent additional degrees of freedom. However, in the quantum theory - where distributional fields are allowed - these fields are interpreted as providing additional, independent degrees of freedom associated with the

Living Reviews in Relativity (2001-6)

http://www. livingreviews.org 
isolated horizon. One then counts the "surface states" of these fields on the isolated horizon subject to a boundary condition relating the surface states to "volume states" and subject to the condition that the area of the isolated horizon (as determined by the volume state) lies within a squared Planck length of the value $A$. This state counting yields an entropy proportional to $A$ for black holes much larger than the Planck scale. Unlike the entanglement entropy and thermal atmosphere calculations, the state counting here yields finite results and no cutoff need be introduced. However, the formula for entropy contains a free parameter (the "Immirzi parameter"), which arises from an ambiguity in the loop quantization procedure, so the constant of proportionality between $S$ and $A$ is not calculable.

The most quantitatively successful calculations of black hole entropy to date are ones arising from string theory. It is believed that at "low energies", string theory should reduce to a 10-dimensional supergravity theory (see [67] for considerable further discussion of the relationship between string theory and 10dimensional and 11-dimensional supergravity). If one treats this supergravity theory as a classical theory involving a spacetime metric, $g_{a b}$, and other classical fields, one can find solutions describing black holes. On the other hand, one also can consider a "weak coupling" limit of string theory, wherein the states are treated perturbatively. In the weak coupling limit, there is no literal notion of a black hole, just as there is no notion of a black hole in linearized general relativity. Nevertheless, certain weak coupling states can be identified with certain black hole solutions of the low energy limit of the theory by a correspondence of their energy and charges. (Here, it is necessary to introduce "D-branes" into string perturbation theory in order to obtain weak coupling states with the desired charges.) Now, the weak coupling states are, in essence, ordinary quantum dynamical degrees of freedom, so their entropy can be computed by the usual methods of statistical physics. Remarkably, for certain classes of extremal and nearly extremal black holes, the ordinary entropy of the weak coupling states agrees exactly with the expression for $A / 4$ for the corresponding classical black hole states; see [58] and [74] for reviews of these results. Recently, it also has been shown [32] that for certain black holes, subleading corrections to the state counting formula for entropy correspond to higher order string corrections to the effective gravitational action, in precise agreement with Eq. (8).

Since the formula for entropy has a nontrivial functional dependence on energy and charges, it is hard to imagine that the agreement between the ordinary entropy of the weak coupling states and black hole entropy could be the result of a random coincidence. Furthermore, for low energy scattering, the absorption/emission coefficients ("gray body factors") of the corresponding weak coupling states and black holes also agree [66]. This suggests that there may be a close physical association between the weak coupling states and black holes, and that the dynamical degrees of freedom of the weak coupling states are likely to at least be closely related to the dynamical degrees of freedom responsible for black hole entropy. However, it remains a challenge to understand in what sense the weak coupling states could be giving an accurate picture of the local physics occurring near (and within) the region classically described as a black

Living Reviews in Relativity (2001-6)

http://www.livingreviews.org 
hole.

The relevant degrees of freedom responsible for entropy in the weak coupling string theory models are associated with conformal field theories. Recently Carlip [33, 34] has attempted to obtain a direct relationship between the string theory state counting results for black hole entropy and the classical Poisson bracket algebra of general relativity. After imposing certain boundary conditions corresponding to the presence of a local Killing horizon, Carlip chooses a particular subgroup of spacetime diffeomorphisms, generated by vector fields $\xi^{a}$. The transformations on the phase space of classical general relativity corresponding to these diffeomorphisms are generated by Hamiltonians $H_{\xi}$. However, the Poisson bracket algebra of these Hamiltonians is not isomorphic to the Lie bracket algebra of the vector fields $\xi^{a}$ but rather corresponds to a central extension of this algebra. A Virasoro algebra is thereby obtained. Now, it is known that the asymptotic density of states in a conformal field theory based upon a Virasoro algebra is given by a universal expression (the "Cardy formula") that depends only on the Virasoro algebra. For the Virasoro algebra obtained by Carlip, the Cardy formula yields an entropy in agreement with Eq. (9). Since the Hamiltonians, $H_{\xi}$, are closely related to the corresponding Noether currents and charges occurring in the derivation of Eqs. (8) and (9), Carlip's approach holds out the possibility of providing a direct, general explanation of the remarkable agreement between the string theory state counting results and the classical formula for the entropy of a black hole.

Living Reviews in Relativity (2001-6)

http://www. livingreviews.org 
The Thermodynamics of Black Holes

\section{Open Issues}

The results described in the previous sections provide a remarkably compelling case that stationary black holes are localized thermal equilibrium states of the quantum gravitational field, and that the laws of black hole mechanics are simply the ordinary laws of thermodynamics applied to a system containing a black hole. Although no results on black hole thermodynamics have been subject to any experimental or observational tests, the theoretical foundation of black hole thermodynamics appears to be sufficiently firm as to provide a solid basis for further research and speculation on the nature of quantum gravitational phenomena. In this section, I will briefly discuss two key unresolved issues in black hole thermodynamics which may shed considerable further light upon quantum gravitational physics.

\subsection{Does a pure quantum state evolve to a mixed state in the process of black hole formation and evaporation?}

In classical general relativity, the matter responsible for the formation of a black hole propagates into a singularity lying within the deep interior of the black hole. Suppose that the matter which forms a black hole possesses quantum correlations with matter that remains far outside of the black hole. Then it is hard to imagine how these correlations could be restored during the process of black hole evaporation unless gross violations of causality occur. In fact, the semiclassical analyses of the Hawking process show that, on the contrary, correlations between the exterior and interior of the black hole are continually built up as it evaporates (see [III] for further discussion). Indeed, these correlations play an essential role in giving the Hawking radiation an exactly thermal character [98].

As already mentioned in subsection 4.1 above, an isolated black hole will "evaporate" completely via the Hawking process within a finite time. If the correlations between the inside and outside of the black hole are not restored during the evaporation process, then by the time that the black hole has evaporated completely, an initial pure state will have evolved to a mixed state, i.e., "information" will have been lost. In a semiclassical analysis of the evaporation process, such information loss does occur and is ascribable to the propagation of the quantum correlations into the singularity within the black hole. A key unresolved issue in black hole thermodynamics is whether this conclusion continues to hold in a complete quantum theory of gravity. On one hand, arguments can be given [III] that alternatives to information loss - such as the formation of a high entropy "remnant" or the gradual restoration of correlations during the late stages of the evaporation process - seem highly implausible. On the other hand, it is commonly asserted that the evolution of an initial pure state to a final mixed state is in conflict with quantum mechanics. For this reason, the issue of whether a pure state can evolve to a mixed state in the process of black hole formation and evaporation is usually referred to as the "black hole information paradox".

Living Reviews in Relativity (2001-6)

http://www.livingreviews.org 
There appear to be two logically independent grounds for the claim that the evolution of an initial pure state to a final mixed state is in conflict with quantum mechanics:

i. Such evolution is asserted to be incompatible with the fundamental principles of quantum theory, which postulates a unitary time evolution of a state vector in a Hilbert space.

ii. Such evolution necessarily gives rise to violations of causality and/or energymomentum conservation and, if it occurred in the black hole formation and evaporation process, there would be large violations of causality and/or energy-momentum (via processes involving "virtual black holes") in ordinary laboratory physics.

With regard to (1), within the semiclassical framework, the evolution of an initial pure state to a final mixed state in the process of black hole formation and evaporation can be attributed to the fact that the final time slice fails to be a Cauchy surface for the spacetime [III]. No violation of any of the local laws of quantum field theory occurs. In fact, a closely analogous evolution of an initial pure state to a final mixed state occurs for a free, massless field in Minkowski spacetime if one chooses the final "time" to be a hyperboloid rather than a hyperplane [III]. (Here, the "information loss" occurring during the time evolution results from radiation to infinity rather than into a black hole.) Indeed, the evolution of an initial pure state to a final mixed state is naturally accommodated within the framework of the algebraic approach to quantum theory [III]] as well as in the framework of generalized quantum theory [5]].

The main arguments for (2) were given in [II] (see also [42]). However, these arguments assume that the effective evolution law governing laboratory physics has a "Markovian" character, so that it is purely local in time. As pointed out in [96], one would expect a black hole to retain a "memory" (stored in its external gravitational field) of its energy-momentum, so it is far from clear that an effective evolution law modeling the process of black hole formation and evaporation should be Markovian in nature. Furthermore, even within the Markovian context, it is not difficult to construct models where rapid information loss occurs at the Planck scale, but negligible deviations from ordinary dynamics occur at laboratory scales [96].

For the above reasons, I do not feel that the issue of whether a pure state evolves to a mixed state in the process of black hole formation and evaporation should be referred to as a "paradox". Nevertheless, the resolution of this issue is of great importance: If pure states remain pure, then our basic understanding of black holes in classical and semiclassical gravity will have to undergo significant revision in quantum gravity. On the other hand, if pure states evolve to mixed states in a fully quantum treatment of the gravitational field, then at least the aspect of the classical singularity as a place where "information can get lost" must continue to remain present in quantum gravity. In that case, rather than "smooth out" the singularities of classical general relativity, one might expect singularities to play a fundamental role in the formulation of quantum

Living Reviews in Relativity (2001-6)

http://www.livingreviews.org 
gravity [76]. Thus, the resolution of this issue would tell us a great deal about both the nature of black holes and the existence of singularities in quantum gravity.

\subsection{What (and where) are the degrees of freedom respon- sible for black hole entropy?}

The calculations described in section 5 yield a seemingly contradictory picture of the degrees of freedom responsible for black hole entropy. In the entanglement entropy and thermal atmosphere approaches, the relevant degrees of freedom are those associated with the ordinary degrees of freedom of quantum fields outside of the black hole. However, the dominant contribution to these degrees of freedom comes from (nearly) Planck scale modes localized to (nearly) a Planck length of the black hole, so, effectively, the relevant degrees of freedom are associated with the horizon. In the quantum geometry approach, the relevant degrees of freedom are also associated with the horizon but appear to have a different character in that they reside directly on the horizon (although they are constrained by the exterior state). Finally the string theory calculations involve weak coupling states, so it is not clear what the degrees of freedom of these weak coupling states would correspond to in a low energy limit where these states may admit a black hole interpretation. However, there is no indication in the calculations that these degrees of freedom should be viewed as being localized near the black hole horizon.

The above calculations are not necessarily in conflict with each other, since it is possible that they each could represent a complementary aspect of the same physical degrees of freedom. Nevertheless, it seems far from clear as to whether we should think of these degrees of freedom as residing outside of the black hole (e.g., in the thermal atmosphere), on the horizon (e.g., in Chern-Simons states), or inside the black hole (e.g., in degrees of freedom associated with what classically corresponds to the singularity deep within the black hole).

The following puzzle [104] may help bring into focus some of the issues related to the degrees of freedom responsible for black hole entropy and, indeed, the meaning of entropy in quantum gravitational physics. As we have already discussed, one proposal for accounting for black hole entropy is to attribute it to the ordinary entropy of its thermal atmosphere. If one does so, then, as previously mentioned in section 5 above, one has the major puzzle of explaining why the quantum field degrees of freedom near the horizon contribute enormously to entropy, whereas the similar degrees of freedom that are present throughout the universe - and are locally indistinguishable from the thermal atmosphere are treated as mere "vacuum fluctuations" which do not contribute to entropy. But perhaps an even greater puzzle arises if we assign a negligible entropy to the thermal atmosphere (as compared with the black hole area, A), as would be necessary if we wished to attribute black hole entropy to other degrees of freedom. Consider a black hole enclosed in a reflecting cavity which has come to equilibrium with its Hawking radiation. Surely, far from the black hole, the thermal atmosphere in the cavity must contribute an entropy given by the usual

Living Reviews in Relativity (2001-6)

http://www.livingreviews.org 
formula for a thermal gas in (nearly) flat spacetime. However, if the thermal atmosphere is to contribute a negligible total entropy (as compared with $A$ ), then at some proper distance $D$ from the horizon much greater than the Planck length, the thermal atmosphere must contribute to the entropy an amount that is much less than the usual result $\left(\propto T^{3}\right)$ that would be obtained by a naive counting of modes. If that is the case, then consider a box of ordinary thermal matter at infinity whose energy is chosen so that its floating point would be less than this distance $D$ from the horizon. Let us now slowly lower the box to its floating point. By the time it reaches its floating point, the contents of the box are indistinguishable from the thermal atmosphere, so the entropy within the box also must be less than what would be obtained by usual mode counting arguments. It follows that the entropy within the box must have decreased during the lowering process, despite the fact that an observer inside the box still sees it filled with thermal radiation and would view the lowering process as having been adiabatic. Furthermore, suppose one lowers (or, more accurately, pushes) an empty box to the same distance from the black hole. The entropy difference between the empty box and the box filled with radiation should still be given by the usual mode counting formulas. Therefore, the empty box would have to be assigned a negative entropy.

I believe that in order to gain a better understanding of the degrees of freedom responsible for black hole entropy, it will be necessary to achieve a deeper understanding of the notion of entropy itself. Even in flat spacetime, there is far from universal agreement as to the meaning of entropy - particularly in quantum theory - and as to the nature of the second law of thermodynamics. The situation in general relativity is considerably murkier [1103] , as, for example, there is no unique, rigid notion of "time translations" and classical general relativistic dynamics appears to be incompatible with any notion of "ergodicity". It seems likely that a new conceptual framework will be required in order to have a proper understanding of entropy in quantum gravitational physics.

Living Reviews in Relativity (2001-6)

http://www.livingreviews.org 


\section{Acknowledgements}

This research was supported in part by NSF grant PHY 95-14726 to the University of Chicago.

Living Reviews in Relativity (2001-6) http://www.livingreviews.org 


\section{References}

[1] Aizenman, M., and Lieb, E.H., "The Third Law of Thermodynamics and the Degeneracy of the Ground State for Lattice Systems", J. Stat. Phys., 24, 279-297, (1981). 2

[2] Anderson, W., "Does the GSL Imply an Entropy Bound?", in Pullin, J., ed., Matters of Gravity. American Physical Society Topical Group in Gravitation, (1999). This issue of the newsletter of the APS Topical Group on Gravitation is available online at http://arxiv.org/abs/gr-qc/9909022 (September, 1999),[Online Los Alamos Archive Preprint]: cited on 6 April 2001. B

[3] Ashtekar, A., Baez, J., Corichi, A., and Krasnov, K., "Quantum Geometry and Black Hole Entropy", Phys. Rev. Lett., 80, 904-907, (1998). For a related online version see: A. Ashtekar, et al., "Quantum Geometry and Black Hole Entropy", (October, 1997), [Online Los Alamos Archive Preprint]: cited on 6 April 2001, http://arxiv.org/abs/ gr-qc/9710007. 5

[4] Ashtekar, A., Beetle, C., Dreyer, O., Fairhurst, S., Krishnan, B., Lewandowski, J., and Wisniewski, J., "Generic Isolated Horizons and their Applications", Phys. Rev. Lett., 85, 3564-3567, (2000). For a related online version see: A. Ashtekar, et al., "Generic Isolated Horizons and their Applications", (June, 2000), [Online Los Alamos Archive Preprint]: cited on 6 April 2001, http://arxiv.org/abs/gr-qc/0006006. 2

[5] Ashtekar, A., Beetle, C., and Fairhurst, S., "Isolated Horizons: A Generalization of Black Hole Mechanics", Class. Quantum Grav., 16, L1L7, (1999). For a related online version see: A. Ashtekar, et al., "Isolated Horizons: A Generalization of Black Hole Mechanics", (December, 1998), [Online Los Alamos Archive Preprint]: cited on 6 April 2001, http://arxiv.org/abs/gr-qc/9812065. 2

[6] Ashtekar, A., Beetle, C., and Fairhurst, S., "Mechanics of Isolated Horizons", Class. Quantum Grav., 17, 253-298, (2000). For a related online version see: A. Ashtekar, et al., "Mechanics of Isolated Horizons", (July, 1999), [Online Los Alamos Archive Preprint]: cited on 6 April 2001, http://arxiv.org/abs/gr-qc/9907068. 2

[7] Ashtekar, A., and Corichi, A., "Laws Governing Isolated Horizons: Inclusion of Dilaton Couplings", Class. Quantum Grav., 17, 1317-1332, (2000). For a related online version see: A. Ashtekar, et al., "Laws Governing Isolated Horizons: Inclusion of Dilaton Couplings", (October, 1999), [Online Los Alamos Archive Preprint]: cited on 6 April 2001, http://arxiv.org/abs/gr-qc/9910068. 2

Living Reviews in Relativity (2001-6)

http://www.livingreviews.org 
[8] Ashtekar, A., Corichi, A., and Krasnov, K., "Isolated Horizons: the Classical Phase Space", Adv. Theor. Math. Phys., 3, 419-478, (2000). For a related online version see: A. Ashtekar, et al., "Isolated Horizons: the Classical Phase Space", (May, 1999), [Online Los Alamos Archive Preprint]: cited on 6 April 2001, http://arxiv.org/abs/gr-qc/9905089. 5

[9] Ashtekar, A., Fairhurst, S., and Krishnan, B., "Isolated Horizons: Hamiltonian Evolution and the First Law", Phys. Rev. D, 62, 104025, (2000). For a related online version see: A. Ashtekar, et al., "Isolated Horizons: Hamiltonian Evolution and the First Law", (May, 2000), [Online Los Alamos Archive Preprint]: cited on 6 April 2001, http://arxiv.org/ abs/gr-qc/0005083. 2, 5

[10] Ashtekar, A., and Krasnov, K., "Quantum Geometry and Black Holes", in Iyer, B.R., and Bhawal, B., eds., Black Holes, Gravitational Radiation, and the Universe, 149-170, (Kluwer Academic Publishers, Dordrecht, 1999). For a related online version see: A. Ashtekar, et al., "Quantum Geometry and Black Holes", (April, 1998), [Online Los Alamos Archive Preprint]: cited on 6 April 2001, http://arxiv.org/abs/ gr-qc/9804039. 5

[11] Banks, T., Susskind, L., and Peskin, M.E., "Difficulties for the Evolution of Pure States into Mixed States", Nucl. Phys. B, 244, 125-134, (1984). 6.

[12] Bardeen, J.M., Carter, B., and Hawking, S.W., "The Four Laws of Black Hole Mechanics", Commun. Math. Phys., 31, 161-170, (1973). 2, 2, 2, 2, 4.1

[13] Bekenstein, J.D., "On Page's examples challenging the entropy bound", (June, 2000), [Online Los Alamos Archive Preprint]: cited on 6 April 2001, http://arxiv.org/abs/gr-qc/0006003. 4.2

[14] Bekenstein, J.D., "Black Holes and Entropy", Phys. Rev. D, 7, 2333-2346, (1973). 2, 4.1, 4.1

[15] Bekenstein, J.D., "Generalized Second Law of Thermodynamics in BlackHole Physics", Phys. Rev. D, 9, 3292-3300, (1974). 2, 4.1, 4.1

[16] Bekenstein, J.D., "Universal Upper Bound on the Entropy-to-Energy Ratio for Bounded Systems", Phys. Rev. D, 23, 287-298, (1981). 4.1, 4.2

[17] Bekenstein, J.D., "Entropy Bounds and the Second Law for Black Holes", Phys. Rev. D, 27, 2262-2270, (1983). 国, 国

[18] Bekenstein, J.D., "Entropy Content and Information Flow in Systems with Limited Energy", Phys. Rev. D, 30, 1669-1679, (1984). 4.2, 4.2

[19] Bekenstein, J.D., "Entropy Bounds and Black Hole Remnants", Phys. Rev. D, 49, 1912-1921, (1994). ii1

Living Reviews in Relativity (2001-6) http://www.livingreviews.org 
[20] Bekenstein, J.D., "Do We Understand Black Hole Entropy ?", in Jantzen, R.T., and Mac Keiser, G., eds., Proceedings of the VII Marcel Grossman Meeting, 39-58, (World Scientific Press, Singapore, 1996). For a related online version see: J.D. Bekenstein, "Do We Understand Black Hole Entropy ?", (September, 1994), [Online Los Alamos Archive Preprint]: cited on 6 April 2001, http://arxiv.org/abs/gr-qc/9409015. 3

[21] Bekenstein, J.D., "Non-Archimedian Character of Quantum Buoyancy and the Generalized Second Law of Thermodynamics", Phys. Rev. D, 60, 124010/9 pages, (1999). For a related online version see: J.D. Bekenstein, "Non-Archimedian character of quantum buoyancy and the generalized second law of thermodynamics", (June, 1999), [Online Los Alamos Archive Preprint]: cited on 6 April 2001, http://arxiv.org/abs/ gr-qc/9906058. iv, 1.2

[22] Bekenstein, J.D., and Schiffer, M., "Quantum Limitations on the Storage and Transmission of Information", Int. J. Mod. Phys. C, 1, 355, (1990). 4.2

[23] Bisognano, J.J., and Wichmann, E.H., "On the Duality Condition for Quantum Fields", J. Math. Phys., 17, 303-321, (1976). 1]

[24] Bombelli, L., Koul, R.K., Lee, J., and Sorkin, R., "Quantum Source of Entropy for Black Holes", Phys. Rev. D, 34, 373-383, (1986). 5

[25] Bousso, R., "A Covariant Entropy Conjecture", J. High Energy Phys., 07, 004, (1999). For a related online version see: R. Bousso, "A Covariant Entropy Conjecture", (May, 1999), [Online Los Alamos Archive Preprint]: cited on 6 April 2001, http://arxiv.org/abs/hep-th/9905177. 3

[26] Bousso, R., "Holography in General Space-times", J. High Energy Phys., 06, 028, (1999). For a related online version see: R. Bousso, "Holography in General Space-times", (June, 1999), [Online Los Alamos Archive Preprint]: cited on 6 April 2001, http://arxiv.org/abs/hep-th/ 990602.2. 目

[27] Bousso, R., "The Holographic Principle for General Backgrounds", Class. Quantum Grav., 17, 997-1005, (2000). For a related online version see: R. Bousso, "The Holographic Principle for General Backgrounds", (November, 1999), [Online Los Alamos Archive Preprint]: cited on 6 April 2001, http://arxiv.org/abs/hep-th/9911002. 3

[28] Brout, R., Massar, S., Parentani, R., and Spindel, Ph., "Hawking Radiation Without Transplanckian Frequencies", Phys. Rev. D, 52, 4559-4568, (1995). For a related online version see: R. Brout, et al., "Hawking Radiation Without Transplanckian Frequencies", (June, 1995), [Online Los Alamos Archive Preprint]: cited on 6 April 2001, http://arxiv.org/ abs/hep-th/9506121. 回

Living Reviews in Relativity (2001-6)

http://www.livingreviews.org 
[29] Brown, J.D., and York, J.W., "Microcanonical Functional Integral for the Gravitational Field", Phys. Rev. D, 47, 1420-1431, (1993). 5

[30] Brown, J.D., and York, J.W., "Quasilocal Energy and Conserved Charges Derived from the Gravitational Action", Phys. Rev. D, 47, 1407-1419, (1993). 4.2

[31] Callen, C., and Wilzcek, F., "On Geometric Entropy", Phys. Lett. B, 333, 55-61, (1994). 5

[32] Cardoso, G.L., de Wit, B., and Mohaupt, T., "Area Law Corrections from State Counting and Supergravity", Class. Quantum Grav., 17, 10071015, (2000). For a related online version see: G.L. Cardoso, et al., "Area Law Corrections from State Counting and Supergravity", (October, 1999), [Online Los Alamos Archive Preprint]: cited on 6 April 2001, http: //arxiv.org/abs/hep-th/9910179. 5

[33] Carlip, S., "Entropy from Conformal Field Theory at Killing Horizons", Class. Quantum Grav., 16, 3327-3348, (1999). For a related online version see: S. Carlip, "Entropy from Conformal Field Theory at Killing Horizons", (June, 1999), [Online Los Alamos Archive Preprint]: cited on 6 April 2001, http://arxiv.org/abs/gr-qc/9906126. 5

[34] Carlip, S., "Black Hole Entropy from Horizon Conformal Field Theory", Nucl. Phys. Proc. Suppl., 88, 10-16, (2000). For a related online version see: S. Carlip, "Black Hole Entropy from Horizon Conformal Field Theory", (December, 1999), [Online Los Alamos Archive Preprint]: cited on 6 April 2001, http://arxiv.org/abs/gr-qc/9912118. 5

[35] Carter, B., "Black Hole Equilibrium States", in DeWitt, C., and DeWitt, B.S., eds., Black Holes, 57-214, (Gordon and Breach, New York, 1973). 2, 2 2

[36] Chrusciel, P.T., Delay, E., Galloway, G.J., and Howard, R., "Regularity of Horizons and The Area Theorem", (January, 2000), [Online Los Alamos Archive Preprint]: cited on 6 April 2001, http://arxiv.org/ abs/gr-qc/0001003. Final version to appear in Annales Henri Poincare. 2

[37] Chrusciel, P.T., and Wald, R.M., "Maximal Hypersurfaces in Stationary Asymptotically Flat Spacetimes", Commun. Math. Phys., 163, 561-604, (1994). 2

[38] Corichi, A., Nucamendi, U., and Sudarsky, D., "Einstein-Yang-Mills Isolated Horizons: Phase Space, Mechanics, Hair and Conjectures", Phys. Rev. D, 62, 044046/19 pages, (2000). For a related online version see: A. Corichi, et al., "Einstein-Yang-Mills Isolated Horizons: Phase Space, Mechanics, Hair and Conjectures", (February, 2000), [Online Los Alamos Archive Preprint]: cited on 6 April 2001, http://arxiv.org/abs/ gr-qc/0002078. 2 
[39] Corley, S., and Jacobson, T., "Hawking Spectrum and High Frequency Dispersion", Phys. Rev. D, 54, 1568-1586, (1996). For a related online version see: S. Corley, et al., "Hawking Spectrum and High Frequency Dispersion", (January, 1996), [Online Los Alamos Archive Preprint]: cited on 6 April 2001, http://arxiv.org/abs/hep-th/9601073. 回

[40] Corley, S., and Jacobson, T., "Lattice Black Holes", Phys. Rev. D, 57, 6269-6279, (1998). For a related online version see: S. Corley, et al., "Lattice Black Holes", (September, 1997), [Online Los Alamos Archive Preprint]: cited on 6 April 2001, http://arxiv.org/abs/hep-th/ 9709166. ए

[41] Dou, D., Causal Sets, a Possible Interpretation for the Black Hole Entropy, and Related Topics, PhD Thesis, (SISSA, Trieste, 1999). 5

[42] Ellis, J., Hagelin, J.S., Nanopoulos, D.V., and Srednicki, M., "Search for Violations of Quantum Mechanics", Nucl. Phys. B, 241, 381-405, (1984). 6.]

[43] Flanagan, E.E., Marolf, D., and Wald, R.M., "Proof of Classical Versions of the Bousso Entropy Bound and of the Generalized Second Law", Phys. Rev. D, 62, 084035/11 pages, (2000). For a related online version see: E.E. Flanagan, et al., "Proof of Classical Versions of the Bousso Entropy Bound and of the Generalized Second Law", (August, 1999), [Online Los Alamos Archive Preprint]: cited on 6 April 2001, http://arxiv.org/ abs/hep-th/9908070. 4.2 , 33

[44] Fredenhagen, K., and Haag, R., "On the Derivation of the Hawking Radiation Associated with the Formation of a Black Hole", Commun. Math. Phys., 127, 273-284, (1990). [1

[45] Friedrich, H., Racz, I., and Wald, R.M., "On the Rigidity Theorem for Spacetimes with a Stationary Event Horizon or a Compact Cauchy Horizon", Commun. Math. Phys., 204, 691-707, (1999). For a related online version see: H. Friedrich, et al., "On the Rigidity Theorem for Spacetimes with a Stationary Event Horizon or a Compact Cauchy Horizon", (November, 1998), [Online Los Alamos Archive Preprint]: cited on 6 April 2001, http://arxiv.org/abs/gr-qc/9811021. 2

[46] Frolov, V.P., and Fursaev, D.V., "Mechanism of Generation of Black Hole Entropy in Sakharov's Induced Gravity", Phys. Rev. D, 56, 2212-2225, (1997). For a related online version see: V.P. Frolov, et al., "Mechanism of Generation of Black Hole Entropy in Sakharov's Induced Gravity", (March, 1997), [Online Los Alamos Archive Preprint]: cited on 6 April 2001, http://arxiv.org/abs/hep-th/9703178. 5

[47] Frolov, V.P., Fursaev, D.V., and Zelnikov, A.I., "Statistical Origin of Black Hole Entropy in Induced Gravity", Nucl. Phys. B, 486, 339-352,

Living Reviews in Relativity (2001-6)

http://www.livingreviews.org 
(1997). For a related online version see: V.P. Frolov, et al., "Statistical Origin of Black Hole Entropy in Induced Gravity", (July, 1996), [Online Los Alamos Archive Preprint]: cited on 6 April 2001, http: //arxiv.org/abs/hep-th/9607104. 5

[48] Frolov, V.P., and Page, D.N., "Proof of the Generalized Second Law for Quasistatic, Semiclassical Black Holes", Phys. Rev. Lett., 71, 3902-3905, (1993). 4.1

[49] Geroch, R., colloquium given at Princeton University, unpublished, (December, 1971). 4.1

[50] Gibbons, G., and Hawking, S.W., "Action Integrals and Partition Functions in Quantum Gravity", Phys. Rev. D, 15, 2752-2756, (1977). 5

[51] Hartle, J., "Generalized Quantum Theory in Evaporating Black Hole Spacetimes", in Wald, R.M., ed., Black Holes andRelativistic Stars, 195219, (University of Chicago Press, Chicago, 1998). For a related online version see: J. Hartle, "Generalized Quantum Theory in Evaporating Black Hole Spacetimes", (May, 1997), [Online Los Alamos Archive Preprint]: cited on 6 April 2001, http://arxiv.org/abs/gr-qc/9705022. 6.1

[52] Hartle, J.B., and Hawking, S.W., "Path Integral Derivation of Black Hole Radiance", Phys. Rev. D, 13, 2188-2203, (1976). 1

[53] Hawking, S.W., "Gravitational Radiationfrom Colliding Black Holes", Phys. Rev. Lett., 26, 1344-1346, (1971). 2

[54] Hawking, S.W., "Particle Creation by Black Holes", Commun. Math.

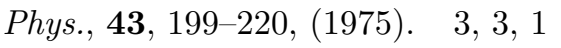

[55] Hawking, S.W., and Ellis, G.F.R., The Large Scale Structure of Spacetime, (Cambridge University Press, Cambridge, 1973). 2, 2

[56] Heusler, M., Black Hole Uniqueness Theorems, (Cambridge University Press, Cambridge, 1996). 2, 2

[57] Holzhey, C., Larsen, F., and Wilzcek, F., "Geometric and Renormalized Entropy in Conformal Field Theory", Nucl. Phys. B, 424, 443-467, (1994). 5

[58] Horowitz, G., "Quantum States of Black Holes", in Wald, R.M., ed., Black Holes and Relativistic Stars, 241-266, (University of Chicago Press, Chicago, 1998). For a related online version see: G. Horowitz, "Quantum States of Black Holes", (April, 1997), [Online Los Alamos Archive Preprint]: cited on 6 April 2001, http://arxiv.org/abs/ gr-qc/9704072. 5

[59] Israel, W., "Third Law of Black-Hole Dynamics: a Formulation and Proof", Phys. Rev. Lett., 57, 397-399, (1986). 2

Living Reviews in Relativity (2001-6)

http://www.livingreviews.org 
[60] Iyer, V., and Wald, R.M., "Some Properties of Noether Charge and a Proposal for Dynamical Black Hole Entropy", Phys. Rev. D, 50, 846-864, (1994). 2

[61] Iyer, V., and Wald, R.M., "A Comparison of Noether Charge and Euclidean Methods for Computing the Entropy of Stationary Black Holes", Phys. Rev. D, 52, 4430-4439, (1995). For a related online version see: V. Iyer, et al., "A Comparison of Noether Charge and Euclidean Methods for Computing the Entropy of Stationary Black Holes", (March, 1995), [Online Los Alamos Archive Preprint]: cited on 6 April 2001, http://arxiv.org/abs/gr-qc/9503052. 5

[62] Jacobson, T., "On the Origin of the Outgoing Black Hole Modes", Phys. Rev. D, 53, 7082-7088, (1996). For a related online version see: T. Jacobson, "On the Origin of the Outgoing Black Hole Modes", (January, 1996), [Online Los Alamos Archive Preprint]: cited on 6 April 2001, http://arxiv.org/abs/hep-th/9601064. [1]

[63] Jacobson, T., and Mattingly, D., "Hawking radiation on a falling lattice", Phys. Rev. D, 61, 024017/10 pages, (2000). For a related online version see: T. Jacobson, et al., "Hawking radiation on a falling lattice", (August, 1999), [Online Los Alamos Archive Preprint]: cited on 6 April 2001, http: //arxiv.org/abs/hep-th/9908099. [1

[64] Kay, B.S., and Wald, R.M., "Theorems on the Uniqueness and Thermal Properties of Stationary, Nonsingular, Quasifree States on Spacetimes with a Bifurcate Killing Horizon", Phys. Rep., 207, 49-136, (1991). 1, 2

[65] Lewandowski, J., "Spacetimes Admitting Isolated Horizons", Class. Quantum Grav., 17, L53-L59, (2000). For a related online version see: J. Lewandowski, "Spacetimes Admitting Isolated Horizons", (July, 1999), [Online Los Alamos Archive Preprint]: cited on 6 April 2001, http: //arxiv.org/abs/gr-qc/9907058. 22

[66] Maldacena, J.M., and Strominger, A., "Black Hole Greybody Factors and D-Brane Spectroscopy", Phys. Rev. D, 55, 861-870, (1997). For a related online version see: J.M. Maldacena, et al., "Black Hole Greybody Factors and D-Brane Spectroscopy", (September, 1996), [Online Los Alamos Archive Preprint]: cited on 6 April 2001, http://arxiv. org/abs/hep-th/9609026. 5

[67] Marolf, D., "String/M-branes for Relativists", (August, 1999), [Online Los Alamos Archive Preprint]: cited on 6 April 2001, http://arxiv. org/abs/gr-qc/9908045. 5

[68] Misner, C.W., Thorne, K.S., and Wheeler, J.A., Gravitation, (Freeman, San Francisco, 1973). 4.2

Living Reviews in Relativity (2001-6)

http://www.livingreviews.org 
[69] Mukohyama, S., "Aspects of black hole entropy", (December, 1999), [Online Los Alamos Archive Preprint]: cited on 6 April 2001, http: //arxiv.org/abs/gr-qc/9912103.

[70] Page, D.N., "Defining Entropy Bounds", (July, 2000), [Online Los Alamos Archive Preprint]: cited on 6 April 2001, http://arxiv.org/abs/ hep-th/0007238. 4.2, 4.2

[71] Page, D.N., "Huge Violations of Bekenstein's Entropy Bound", (May, 2000), [Online Los Alamos Archive Preprint]: cited on 6 April 2001, http://arxiv.org/abs/gr-qc/0005111. 4.2

[72] Page, D.N., "Subsystem Entropy Exceeding Bekenstein's Bound", (July, 2000), [Online Los Alamos Archive Preprint]: cited on 6 April 2001, http: //arxiv.org/abs/hep-th/0007237. 4.2

[73] Parker, L., "Quantized Fields and Particle Creation in Expanding Universes", Phys. Rev., 183, 1057-1068, (1969). 3

[74] Peet, A., "TASI lectures on black holes in string theory", (August, 2000), [Online Los Alamos Archive Preprint]: cited on 6 April 2001, http: //arxiv.org/abs/hep-th/0008241. 5

[75] Pelath, M.A., and Wald, R.M., "Comment on Entropy Bounds and the Generalized Second Law", Phys. Rev. D, 60, 104009/4 pages, (1999). For a related online version see: M.A. Pelath, et al., "Comment on entropy bounds and the generalized second law", (January, 1999), [Online Los Alamos Archive Preprint]: cited on 6 April 2001, http://arxiv.org/ abs/gr-qc/9901032. 4.2

[76] Penrose, R., "Singularities and Time-Asymmetry", in Hawking, S.W., and Israel, W., eds., General Relativity, an Einstein Centennary Survey, 581638, (Cambridge University Press, Cambridge, 1979). 6.1

[77] Penrose, R., "Quasi-Local Mass and Angular Momentum", Proc. R. Soc. London, A381, 53-63, (1982). 4.2

[78] Racz, I., and Wald, R.M., "Global Extensions of Spacetimes Describing Asymptotic Final States of Black Holes", Class. Quantum Grav., 13, 539552, (1996). For a related online version see: I. Racz, et al., "Global Extensions of Spacetimes Describing Asymptotic Final States of Black Holes", (July, 1995), [Online Los Alamos Archive Preprint]: cited on 6 April 2001, http://arxiv.org/abs/gr-qc/9507055. 2, (1)

[79] Reznik, B., "Trans-Planckian Tail in a Theory with a Cutoff", Phys. Rev. $D, 55,2152-2158$, (1997). For a related online version see: B. Reznik, "Trans-Planckian Tail in a Theory with a Cutoff", (June, 1996), [Online Los Alamos Archive Preprint]: cited on 6 April 2001, http://arxiv. org/abs/gr-qc/9606083. [] 
[80] Sorkin, R., "Two Topics Concerning Black Holes: Extremality of the Energy, Fractality of the Horizon", in Fulling, S.A., ed., Proceedings of the Conference on Heat Kernel Techniques and Quantum Gravity, 387-407, (University of Texas Press, Austin, 1995). For a related online version see: R. Sorkin, "Two Topics Concerning Black Holes: Extremality of the Energy, Fractality of the Horizon", (August, 1995), [Online Los Alamos Archive Preprint]: cited on 6 April 2001, http: 7/arxiv.org/abs/gr-qc/9508002. 2

[81] Sorkin, R., "How Wrinkled is the Surface of a Black Hole?", in Wiltshire, D., ed., Proceedings of the First Australasian Conference on General Relativity and Gravitation, 163-174, (University of Adelaide Press, Adelaide, 1996). For a related online version see: R. Sorkin, "How Wrinkled is the Surface of a Black Hole?", (January, 1997), [Online Los Alamos Archive Preprint]: cited on 6 April 2001, http://arxiv.org/ abs/gr-qc/9701056. 5

[82] Sorkin, R.D., "The Statistical Mechanics of Black Hole Thermodynamics", in Wald, R.M., ed., Black Holes and Relativistic Stars, 177-194, (University of Chicago Press, Chicago, 1998). For a related online version see: R.D. Sorkin, "The Statistical Mechanics of Black Hole Thermodynamics", (May, 1997), [Online Los Alamos Archive Preprint]: cited on 6 April 2001, http://arxiv.org/abs/gr-qc/9705006. 4.1

[83] Sorkin, R.D., Wald, R.M., and Zhang, Z.J., "Entropy of Self-Gravitating Radiation", Gen. Relativ. Gravit., 13, 1127-1146, (1981). 4.2

[84] Sudarsky, D., and Wald, R.M., "Extrema of Mass, Stationarity and Staticity, and Solutions to the Einstein-Yang-Mills Equations", Phys. Rev. D, 46, 1453-1474, (1992). 2, 2

[85] Sudarsky, D., and Wald, R.M., "Mass Formulas for Stationary EinsteinYang-Mills Black Holes and a Simple Proof of Two Staticity Theorems", Phys. Rev. D, 47, R5209-R5213, (1993). 2

[86] Susskind, L., "The World as a Hologram", J. Math. Phys., 36, 63776396, (1995). For a related online version see: L. Susskind, "The World as a Hologram", (September, 1994), [Online Los Alamos Archive Preprint]: cited on 6 April 2001, http://arxiv.org/abs/hep-th/9409089. 3, 3]

[87] Susskind, L., and Uglam, J., "Black Hole Entropy in Canonical Quantum Gravity and Superstring Theory", Phys. Rev. D, 50, 2700-2711, (1994). 回

[88] 't Hooft, G., "On the Quantum Structure of a Black Hole", Nucl. Phys. $B, \mathbf{2 5 6}, 727-745,(1985)$. 5

[89] 't Hooft, G., "On the Quantization of Space and Time", in Markov, M.A., Berezin, V.A., and Frolov, V.P., eds., Quantum Gravity, 551-567, (World Scientific Press, Singapore, 1988). 3

Living Reviews in Relativity (2001-6)

http://www.livingreviews.org 
[90] Thorne, K.S., Zurek, W.H., and Price, R.H., "The Thermal Atmosphere of a Black Hole", in Thorne, K.S., Price, R.H., and Macdonald, D.A., eds., Black Holes: The Membrane Paradigm, 280-340, (Yale University Press, New Haven, 1986). 4.1

[91] Unruh, W.G., "Notes on Black Hole Evaporation", Phys. Rev. D, 14, 870-892, (1976). 目, 目

[92] Unruh, W.G., "Experimental Black-Hole Evaporation?", Phys. Rev. Lett., 46, 1351-1353, (1981). 1

[93] Unruh, W.G., "Dumb Holes and the Effects of High Frequencies on Black Hole Evaporation", Phys. Rev. D, 51, 2827-2838, (1995). For a related online version see: W.G. Unruh, "Dumb Holes and the Effects of High Frequencies on Black Hole Evaporation", (September, 1994), [Online Los Alamos Archive Preprint]: cited on 6 April 2001, http://arxiv.org/ abs/gr-qc/9409008. [1]

[94] Unruh, W.G., and Wald, R.M., "Acceleration Radiation and the Generalized Second Law of Thermodynamics", Phys. Rev. D, 25, 942-958, (1982). 1.1, 1.2

[95] Unruh, W.G., and Wald, R.M., "Entropy Bounds, Acceleration Radiation and the Generalized Second Law", Phys. Rev. D, 27, 2271-2276, (1983). 4.2

[96] Unruh, W.G., and Wald, R.M., "On Evolution Laws Taking Pure States to Mixed States in Quantum Field Theory", Phys. Rev. D, 52, 21762182, (1995). For a related online version see: W.G. Unruh, et al., "On Evolution Laws Taking Pure States to Mixed States in Quantum Field Theory", (March, 1995), [Online Los Alamos Archive Preprint]: cited on 6 April 2001, http://arxiv.org/abs/hep-th/9503024. 6.1

[97] Visser, M., "Hawking radiation without black hole entropy", Phys. Rev. Lett., 80, 3436-3439, (1998). For a related online version see: M. Visser, "Hawking radiation without black hole entropy", (December, 1997), [Online Los Alamos Archive Preprint]: cited on 6 April 2001, http: 7/arxiv.org/abs/gr-qc/9712016. 四

[98] Wald, R.M., "On Particle Creation by Black Holes", Commun. Math. Phys., 45, 9-34, (1975). 3, 1, 6.1

[99] Wald, R.M., General Relativity, (University of Chicago Press, Chicago, 1984). 1, 2, 2

[100] Wald, R.M., "Black Hole Entropy is the Noether Charge", Phys. Rev. D, 48, R3427-R3431, (1993). 5

Living Reviews in Relativity (2001-6)

http://www.livingreviews.org 
[101] Wald, R.M., Quantum Field Theory in Curved Spacetime and Black Hole Thermodynamics, (University of Chicago Press, Chicago, 1994). 2, 3, 1, 4.1, 3, [6.1, 6.1]

[102] Wald, R.M., "'Nernst Theorem' and Black Hole Thermodynamics", Phys. Rev. D, 56, 6467-6474, (1997). For a related online version see: R.M. Wald, "Nernst Theorem' and Black Hole Thermodynamics", (April, 1997), [Online Los Alamos Archive Preprint]: cited on 6 April 2001, http://arxiv.org/abs/gr-qc/9704008. 2

[103] Wald, R.M., "Black Holes and Thermodynamics", in Wald, R.M., ed., Black Holes and Relativistic Stars, 155-176, (University of Chicago Press, Chicago, 1998). For a related online version see: R.M. Wald, "Black Holes and Thermodynamics", (February, 1997), [Online Los Alamos Archive Preprint]: cited on 6 April 2001, http://arxiv.org/abs/ gr-qc/9702022. 6.2

[104] Wald, R.M., "Gravitation, Thermodynamics, and Quantum Theory", Class. Quantum Grav., 16, A177-A190, (1999). For a related online version see: R.M. Wald, "Gravitation, Thermodynamics, and Quantum Theory", (January, 1999), [Online Los Alamos Archive Preprint]: cited on 6 April 2001, http://arxiv.org/abs/gr-qc/9901033. 6.2

[105] Zurek, W.H., and Thorne, K.S., "Statistical Mechanical Origin of the Entropy of a Rotating, Charged Black Hole", Phys. Rev. Lett., 54, 2171$2175,(1986)$. 4.1

Living Reviews in Relativity (2001-6)

http://www.livingreviews.org 